\title{
ON ETA-EINSTEIN SASAKIAN GEOMETRY
}

\author{
CHARLES P. BOYER, KRZYSZTOF GALICKI AND PAOLA MATZEU
}

\begin{abstract}
A compact quasi-regular Sasakian manifold $M$ is foliated by onedimensional leaves and the transverse space of this characteristic foliation is necessarily a compact Kähler orbifold $\mathcal{Z}$. In the case when the transverse space $\mathcal{Z}$ is also Einstein the corresponding Sasakian manifold $M$ is said to be Sasakian $\eta$-Einstein. In this article we study $\eta$-Einstein geometry as a class of distinguished Riemannian metrics on contact metric manifolds. In particular, we use a previous solution of the Calabi problem in the context of Sasakian geometry to prove the existence of $\eta$-Einstein structures on many different compact manifolds, including exotic spheres. We also relate these results to the existence of Einstein-Weyl and Lorenzian Sasakian-Einstein structures.
\end{abstract}

\section{INTRODUCTION}

The purpose of this paper is to study a special kind of Riemannian metric on Sasakian manifolds. A Sasakian manifold $M$ of dimension $2 n+1$ with a Sasakian structure $\mathcal{S}=(\xi, \eta, \Phi, g)$ is said to be $\eta$-Einstein if the Ricci curvature tensor of the metric $g$ satisfies the equation $\operatorname{Ric}_{g}=\lambda g+\nu \eta \otimes \eta$ for some constants $\lambda, \nu \in \mathbb{R}$. These metrics were introduced and studied by Okumura Oku62 and then named by Sasaki Sas65] in his lecture notes in 1965. Okumura assumed that both $\lambda$ and $\nu$ are functions, and then proved, similar to the case of Einstein metrics, that they must be constant when $n>1$. Obviously $\nu=0$ reduces to the more familiar Sasakian-Einstein condition. In general, $\lambda+\nu=2 n$ and every Sasakian $\eta$-Einstein manifold is necessarily of constant scalar curvature $s=2 n(1+\lambda)$.

It is well-known that Sasakian-Einstein metrics are necessarily positive with Einstein constant equal to $\operatorname{dim}(M)-1$. On the other hand, on Kähler manifolds one naturally considers Kähler-Einstein metrics with Einstein constant of any sign. As every Sasakian manifold comes with a characteristic foliation $\mathcal{F}_{\xi}$ whose transverse geometry is Kähler, the notion of the basic first Chern class $c_{1}^{B}$ leads one to define null, positive, or negative Sasakian structures in analogy with Kähler geometry. One simply asks that $c_{1}^{B}$ either vanish or be represented by a basic positive or negative $(1,1)$-form, respectively. In such a context Sasakian $\eta$-Einstein metrics are the natural analogues of Kähler-Einstein metrics.

In the $c_{1}^{B}>0$ case the transverse geometry is Fano and the $\eta$-Einstein condition implies further that the transverse geometry is Kähler-Einstein of positive scalar curvature. It was first observed by Tanno that a positive Sasakian manifold $M$ with an $\eta$-Einstein metric $g$ admits another metric $g^{\prime}$ which is Sasakian-Einstein Tan79. The respective Sasakian structures $\mathcal{S}$ and $\mathcal{S}^{\prime}$ are related by the so-called

1991 Mathematics Subject Classification. Primary 53C25.

Key words and phrases. Sasakian geometry, Einstein-Weyl geometry, Kähler-Einstein metrics, Calabi problem, orbifolds, contact structures. 
'transverse' or 'D -homothety' transformation (see Section 3 for precise definition). Tanno used this simple observation to show that a unit tangent bundle $M=T_{1} S^{n}$ of any $n$-sphere admits a homogeneous Sasakian-Einstein structure. Actually, transverse homotheties define a family of Sasakian $\eta$-Einstein structures for each scale $a \in \mathbb{R}^{+}$. This family can be represented by the unique Sasakian-Einstein structure which then can be either "squashed" or "stretched". It turns out that the squashed Sasakian $\eta$-Einstein structures can be used to define an associated Einstein-Weyl geometry in a canonical way Nar93, Nar97, Nar98, Hig93 PS93. For instance, in 3 dimensions the only compact simply connected Sasakian-Einstein manifold is the round $S^{3}$ and, hence, the squashed $\eta$-Einstein metric is simply the Berger metric. The latter is a well-known example of a non-trivial Einstein-Weyl manifold. It follows that Sasakian-Einstein geometry offers quite a powerful tool in constructing odd-dimensional examples of Einstein-Weyl manifolds. In particular, many compact spin 5-manifolds admit families of Einstein-Weyl structures. Similarly, both the standard as well as some exotic spheres in odd dimensions also admit EinsteinWeyl structures.

The $c_{1}^{B}=0$ case is an example of the so-called transverse Calabi-Yau structure. As a consequence of a transverse version EKA90 of Yau's famous theorem, every null Sasakian structure can be deformed to a Sasakian $\eta$-Einstein structure which is transverse Calabi-Yau. We exhibit interesting examples of such structures on certain compact Sasakian manifolds. Interestingly, some of these examples are also Einstein-Weyl as observed by Narita in Nar93.

In the $c_{1}^{B}<0$ case an orbifold version of the theorem of Aubin and Yau shows that every negative Sasakian structure $\mathcal{S}$ can be deformed to a Sasakian $\eta$-Einstein structure. As a consequence, negative Sasakian $\eta$-Einstein metrics can be easily found on many compact contact spin manifolds. Remarkably, it follows that (possibly all) homotopy spheres which bound parallelizable manifolds admit infinitely many inequivalent negative Sasakian $\eta$-Einstein structures. Hence, for example, $S^{5}$ admits both positive and negative Sasakian $\eta$-Einstein structures, but no null Sasakian structures. Both positive and negative Sasakian $\eta$-Einstein structures exist on many compact simply connected spin 5-manifolds, including $\# k\left(S^{2} \times S^{3}\right)$ for certain $k$ (positive Sasakian structures exist for all $k$ ) and certain rational homology 5-spheres. Some of these manifolds, for example \#20( $\left.S^{2} \times S^{3}\right)$ admit all three types of Sasakian $\eta$-Einstein structures. One additional interesting feature of the $c_{1}^{B}<0$ case is that every negative Sasakian $\eta$-Einstein structure yields a family of Lorentzian Sasakian $\eta$-Einstein metrics Bau00, Boh03. The metrics in the family are related by a transverse homothety and, like in the positive case, there is a unique Lorentzian Sasakian-Einstein metric in the family with Einstein constant $1-\operatorname{dim}(M)$. Hence, for instance, $S^{5}$ admits infinitely many Lorentzian Sasakian-Einstein structures.

By virtue of admitting real Killing spinors, Sasakian-Einstein manifolds have recently received a lot of attention in physics. The physicists' interest in five and seven-dimensional manifolds admitting real Killing spinors dates back to the early eighties, when Kaluza-Klein models played a central role in the supergravity theory. Today's renewed interest in these manifolds has to do with the so-called $p$-brane solutions in superstring theory. These $p$-branes, "near the horizon" are modeled by the pseudo-Riemannian geometry of the product $\operatorname{adS}_{p+2} \times M$, where $\operatorname{adS}_{p+2}$ is the $(p+2)$-dimensional anti-de-Sitter space (a Lorentzian version of a space of constant 
sectional curvature) and $(M, g)$ is a Riemannian manifold of dimension $d=D-p-2$. Here $D$ is the dimension of the original supersymmetric theory. In the most interesting cases of M2-branes, M5-branes, and D3-branes $D$ equals to either 11 (Mp-branes of M-theory) or 10 ( $\mathrm{D} p$-branes in type IIA or type IIB string theory). Any residual supersymmetry forces $M$ to admit real Killing spinors. For example, the case of D3-branes of string theory the relevant near horizon geometry is that of $\operatorname{adS}_{5} \times M$, where $M$ is a Sasakian-Einstein 5-manifold. The D3-brane solution interpolates between adS $\mathrm{S}_{5} \times M$ and $\mathbb{R}^{3,1} \times \mathcal{C}(M)$, where the cone $\mathcal{C}(M)$ is a Calabi-Yau threefold. In its original version the Maladacena conjecture (also known as AdS/CFT duality) states that the 't Hooft large $n$ limit of $N=4$ supersymmetric Yang-Mills theory with gauge group $S U(n)$ is dual to type IIB superstring theory on adS ${ }_{5} \times S^{5}$ Mal99. This conjecture has now been examined for many known Sasakian-Einstein metric in dimension 5 and 7. All this has led to a remarkable discovery of new cohomogeneity one Sasakian-Einstein metrics GMSW04a, GMSW04b. In dimension 5 the new examples produce infinitely many toric Sasakian-Einstein metric on $S^{2} \times S^{3}$. The homogeneous Sasakian-Einstein metric on $S^{2} \times S^{3}$ examined in the context of the AdS/CFT duality by Klebanov and Witten KW99 emerges as a special case of this construction MS05, MSY05. These metrics are of equal if not greater interest from the geometric point of view. They provide the first examples of compact Sasakian-Einstein manifolds which are not quasi-regular, i.e., the space of leaves of the associated characteristic foliation is not a topologically nicely behaved object such as an orbifold. As a consequence they are also counterexamples to a conjecture made in 1994 by Cheeger and Tian [CT94.

Deformations of Sasakian-Einstein metrics naturally lead to Einstein-Weyl structures are important in general relativity theory. Null and negative Sasakian $\eta$ Einstein manifolds have not perhaps received as much attention. Yet, both in physics and in mathematics, they often emerge in a somewhat implicit fashion. For example, null Sasakian manifolds are orbifold bundles over compact CalabiYau orbifolds. They are very natural odd-dimensional companions of the CalabiYau spaces. Hence, for example, 7-dimensional null Sasakian $\eta$-Einstein manifolds should be important in the study of Calabi-Yau 3-folds with cyclic orbifold singularities. In particular all problems regarding famous Mirror Symmetry should have a translation in the language of null Sasakian $\eta$-Einstein 7 -manifolds.

Our article is organized as follows: In Section 2 we recall some basic properties of the transverse geometry including invariants of the characteristic foliation. Section 3 follows with fundamentals about certain deformations of Sasakian structures. In section 4 we begin the study of $\eta$-Einstein metrics and their relation to the Sasakian Calabi problem. Here we also describe the non-spin obstruction to the existence of $\eta$-Einstein metrics. The following section describes some general properties of Sasakian $\eta$-Einstein manifolds, in particular, we describe the relation between negative Sasakian $\eta$-Einstein structures and Lorentzian Sasakian-Einstein structures. Many of the most interesting examples of positive, null, and negative Sasakian structures can be found on links of isolated hypersurface singularities which are discussed in Section 6. In Sections 7 and 8 we use this fact to introduce a large set of examples of Sasakian $\eta$-Einstein structures. Finally, in the last section we discuss relations between Einstein-Weyl and Sasakian $\eta$-Einstein structures.

Acknowledgments . We thank Gang Tian for answering several questions. We would also like to thank János Kollár for numerous discussions and for explaining 
some of his recent results Kol04a to us, and to Liviu Ornea for pointing out reference GO98. CPB and KG were partially supported by the NSF under grant number DMS-0203219. The authors would also like to thank Università di Roma "La Sapienza" for partial support where discussions on this work initiated. The second named author would like to thank Max-Planck-Institute in Bonn for hospitality and support. Much of the work on this article was done during his stay there.

\section{The Characteristic Foliation and Transverse Invariants}

Recall that an almost contact structure on a differentiable manifold $M$ is a triple $(\xi, \eta, \Phi)$, where $\Phi$ is a tensor field of type $(1,1)$ (i.e., an endomorphism of $T M), \xi$ is a vector field, and $\eta$ is a 1 -form which satisfy

$$
\eta(\xi)=1 \text { and } \Phi \circ \Phi=-\mathbb{I}+\xi \otimes \eta,
$$

where $\mathbb{I}$ is the identity endomorphism on $T M$. A smooth manifold with such a structure is called an almost contact manifold. A Riemannian metric $g$ on $M$ is said to be compatible with the almost contact structure if for any vector fields $X, Y$ on $M$ we have

$$
g(\Phi X, \Phi Y)=g(X, Y)-\eta(X) \eta(Y) .
$$

An almost contact structure with a compatible metric is called an almost contact metric structure. In case $\eta$ is a contact form $(\xi, \eta, \Phi, g)$ is said to be a contact metric structure on $M$.

A contact metric structure $(\xi, \eta, \Phi, g)$ is called $K$-contact if $\xi$ is a Killing vector field of $g$ and it is called Sasakian if the metric cone $\left(C(M), d r^{2}+r^{2} g, d\left(r^{2} \eta\right)\right)$ is Kähler.

In this section we study the transverse geometry of the Riemannian foliation $\mathcal{F}_{\xi}$ defined on $M$ by the characteristic, or as it is called Reeb vector field $\xi$. The transverse geometry of Sasakian structures were discussed in part 3 of Sasaki's notes Sas68 in the regular case. This predated the modern development of foliation theory which is the proper setting for the study of Sasakian geometry. We first make note of some well-known properties. The foliation $\mathcal{F}_{\xi}$ is one dimensional whose leaves are geodesics with respect to the Sasakian metric $g$, and this metric is bundle-like.

Let $(M, \xi, \eta, \Phi, g)$ be a Sasakian manifold, and consider the contact subbundle $\mathcal{D}=$ ker $\eta$. There is an orthogonal splitting of the tangent bundle as

$$
T M=\mathcal{D} \oplus L_{\xi},
$$

where $L_{\xi}$ is the trivial line bundle generated by the Reeb vector field $\xi$. The contact subbundle $\mathcal{D}$ is just the normal bundle to the characteristic foliation $\mathcal{F}_{\xi}$ generated by $\xi$. It is naturally endowed with both a complex structure $J=\Phi \mid \mathcal{D}$ and a symplectic structure $d \eta$. Hence, $(\mathcal{D}, J, d \eta)$ gives $M$ a transverse Kähler structure with Kähler form $d \eta$ and metric $g_{\mathcal{D}}$ defined by

$$
g_{\mathcal{D}}(X, Y)=d \eta(X, J Y)
$$

which is related to the Sasakian metric $g$ by

$$
g=g_{\mathcal{D}} \oplus \eta \otimes \eta \text {. }
$$


Recall, see e.g. [Ton97, that a smooth p-form $\alpha$ on $M$ is called basic if

$$
\xi\rfloor \alpha=0, \quad \mathcal{L}_{\xi} \alpha=0,
$$

and we let $\Lambda_{B}^{p}$ denote the sheaf of germs of basic p-forms on $M$, and by $\Omega_{B}^{p}$ the set of global sections of $\Lambda_{B}^{p}$ on $M$. The sheaf $\Lambda_{B}^{p}$ is a module under the ring, $\Lambda_{B}^{0}$, of germs of smooth basic functions on $M$. We let $C_{B}^{\infty}(M)=\Omega_{B}^{0}$ denote global sections of $\Lambda_{B}^{0}$, i.e. the ring of smooth basic functions on $M$. Since exterior differentiation preserves basic forms we get a de Rham complex

$$
\cdots \longrightarrow \Omega_{B}^{p} \stackrel{d}{\longrightarrow} \Omega_{B}^{p+1} \longrightarrow \cdots
$$

whose cohomology $H_{B}^{*}\left(\mathcal{F}_{\xi}\right)$ is called the basic cohomology of $\left(M, \mathcal{F}_{\xi}\right)$. The basic cohomology ring $H_{B}^{*}\left(\mathcal{F}_{\xi}\right)$ is an invariant of the foliation $\mathcal{F}_{\xi}$ and hence, of the Sasakian structure on $M$. When $M$ is compact it is related to the ordinary de Rham cohomology $H^{*}(M, \mathbb{R})$ by the long exact sequence, (see e.g. Ton97])

(6)

$$
\cdots \longrightarrow H_{B}^{p}\left(\mathcal{F}_{\xi}\right) \longrightarrow H^{p}(M, \mathbb{R}) \stackrel{j_{p}}{\longrightarrow} H_{B}^{p-1}\left(\mathcal{F}_{\xi}\right) \stackrel{\delta}{\longrightarrow} H_{B}^{p+1}\left(\mathcal{F}_{\xi}\right) \longrightarrow \cdots
$$

where $\delta$ is the connecting homomorphism given by $\delta[\alpha]=[d \eta \wedge \alpha]=[d \eta] \cup[\alpha]$, and $j_{p}$ is the composition of the map induced by $\left.\xi\right\rfloor$ with the well known isomorphism $H^{r}(M, \mathbb{R}) \approx H^{r}(M, \mathbb{R})^{\mathfrak{T}}$ where $\mathfrak{T}$, a torus, is the closure of the Reeb flow, and $H^{r}(M, \mathbb{R})^{\mathfrak{T}}$ is the $\mathfrak{T}$-invariant cohomology defined from the $\mathfrak{T}$-invariant $\mathrm{r}$-forms $\Omega^{r}(M)^{\mathfrak{T}}$. We also note that $d \eta$ is basic even though $\eta$ is not.

Next we exploit the fact that the transverse geometry is Kähler. Let $\mathcal{D}_{\mathbb{C}}$ denote the complexification of $\mathcal{D}$, and decompose it into its eigenspaces with respect to $J$, that is, $\mathcal{D}_{\mathbb{C}}=\mathcal{D}^{1,0} \oplus \mathcal{D}^{0,1}$. Similarly, we get a splitting of the complexification of the sheaf $\Lambda_{B}^{1}$ of basic one forms on $M$, namely

$$
\Lambda_{B}^{1} \otimes \mathbb{C}=\Lambda_{B}^{1,0} \oplus \Lambda_{B}^{0,1} .
$$

We let $\mathcal{E}_{B}^{p, q}$ denote the sheaf of germs of basic forms of type $(p, q)$, and we obtain a splitting

$$
\Lambda_{B}^{r} \otimes \mathbb{C}=\bigoplus_{p+q=r} \mathcal{E}_{B}^{p, q} .
$$

The basic cohomology groups $H_{B}^{p, q}\left(\mathcal{F}_{\xi}\right)$ are fundamental invariants of a Sasakian structure which enjoy many of the same properties as the ordinary Dolbeault cohomology of a Kähler structure. The following theorem was given in BGN03b:

Theorem 1. Let $(M, \xi, \eta, \Phi, g)$ be a compact Sasakian manifold of dimension $2 n+1$. Then we have

(1) $H_{B}^{n, n}\left(\mathcal{F}_{\xi}\right) \approx \mathbb{R}$.

(2) The class $[d \eta]_{B} \neq 0$ lies in $H_{B}^{1,1}\left(\mathcal{F}_{\xi}\right)$.

(3) $H_{B}^{p, p}\left(\mathcal{F}_{\xi}\right)>0$.

(4) $H_{B}^{2 p+1}\left(\mathcal{F}_{\xi}\right)$ has even dimension for $p<[n / 2]$.

(5) $H^{1}(M, \mathbb{R}) \approx H_{B}^{1}\left(\mathcal{F}_{\xi}\right)$.

(6) (Transverse Hodge Decomposition) $H_{B}^{r}\left(\mathcal{F}_{\xi}\right)=\bigoplus_{p+q=r} H_{B}^{p, q}\left(\mathcal{F}_{\xi}\right)$.

(7) Complex conjugation induces an anti-linear isomorphism

$$
H_{B}^{p, q}\left(\mathcal{F}_{\xi}\right) \approx H_{B}^{q, p}\left(\mathcal{F}_{\xi}\right)
$$


(8) (Transverse Serre Duality) There is an isomorphism

$$
H^{p, q}\left(\mathcal{F}_{\xi}\right) \approx H^{n-p, n-q}\left(\mathcal{F}_{\xi}\right) .
$$

Theorem 1] gives rise to fundamental invariants BGN03c] of the Sasakian structure, namely the basic Betti numbers and basic Hodge numbers:

$$
b_{r}^{B}\left(\mathcal{F}_{\xi}\right)=\operatorname{dim} H_{B}^{r}\left(\mathcal{F}_{\xi}\right) \quad h_{B}^{p, q}\left(\mathcal{F}_{\xi}\right)=\operatorname{dim} H_{B}^{p, q}\left(\mathcal{F}_{\xi}\right)
$$

which are related to the basic Betti numbers by

$$
b_{r}^{B}\left(\mathcal{F}_{\xi}\right)=\sum_{p+q=r} h^{p, q}\left(\mathcal{F}_{\xi}\right),
$$

and satisfy

$$
h_{B}^{p, q}\left(\mathcal{F}_{\xi}\right)=h_{B}^{q, p}\left(\mathcal{F}_{\xi}\right), \quad h_{B}^{p, q}\left(\mathcal{F}_{\xi}\right)=h_{B}^{n-p, n-q}\left(\mathcal{F}_{\xi}\right)
$$

For a description of other transverse holomorphic invariants and relationships between them, we refer the reader to BGN03b BGN03a. Here we only mention the basic geometric genus $p_{g}\left(\mathcal{F}_{\xi}\right)=h_{B}^{0, n}\left(\mathcal{F}_{\xi}\right)$, and the basic irregularity $q=q(\mathcal{S})=h_{B}^{0,1}$. We remark that $q=\frac{1}{2} b_{1}^{B}\left(\mathcal{F}_{\xi}\right)=\frac{1}{2} b_{1}(M)$ is actually a topological invariant by (5) of Theorem 1 In the case $n=2$, things simplify much more, and all the basic Hodge numbers are given in terms of the three invariants $q, p_{g}\left(\mathcal{F}_{\xi}\right)$ and $h_{B}^{1,1}\left(\mathcal{F}_{\xi}\right)$. Recall now

Definition 2. The characteristic foliation $\mathcal{F}_{\xi}$ is said to be quasi-regular if there is a positive integer $k$ such that each point has a foliated coordinate chart $(U, x)$ such that each leaf of $\mathcal{F}_{\xi}$ passes through $U$ at most $k$ times. If $k=1$ then the foliation is called regular. If $\mathcal{F}_{\xi}$ is not quasi-regular, it is said to be irregular. We use the term non-regular to mean quasi-regular but not regular.

In the case of a compact quasi-regular Sasakian manifold $M$ we know that the space of leaves is a compact Riemannian orbifold $\mathcal{Z}$. But since the transverse geometry on $M$ is Kähler, the orbifold must be Kähler. But actually in the quasi-regular case more is true. It follows BG00 that $M$ is the total space of a $\mathrm{V}$-bundle over $\mathcal{Z}$, and the curvature of the connection form $\eta$ is precisely the pullback of the Kähler form on $\mathcal{Z}$. Thus, $\mathcal{Z}$ satisfies an orbifold integrality condition which we now elaborate. This integrality condition ties Sasakian geometry on compact manifolds to projective algebraic geometry. Recall that a compact $\operatorname{Kähler~orbifold~}(\mathcal{Z}, \omega)$ is called a Hodge orbifold if $[\omega]$ lies in $H_{\text {orb }}^{2}(\mathcal{Z}, \mathbb{Z})$. By a theorem of Baily [Bai57] a Hodge orbifold is a polarized projective algebraic variety. We have

Theorem 3. Let $\mathcal{S}=(\xi, \eta, \Phi, g)$ be a compact quasi-regular Sasakian structure on a smooth manifold of dimension $2 n+1$, and let $\mathcal{Z}$ denote the space of leaves of the characteristic foliation. Then

(1) The leaf space $\mathcal{Z}$ is a Hodge orbifold with Kähler metric $h$ and Kähler form $\omega$ which defines an integral class $[\omega]$ in $H_{\text {orb }}^{2}(\mathcal{Z}, \mathbb{Z})$ in such a way that $\pi:(\mathcal{S}, g) \longrightarrow(\mathcal{Z}, h)$ is an orbifold Riemannian submersion. The fibers of $\pi$ are totally geodesic submanifolds of $\mathcal{S}$ diffeomorphic to $S^{1}$.

(2) $\mathcal{Z}$ is also a $\mathbb{Q}$-factorial, polarized, normal projective algebraic variety.

Conversely, given a Hodge orbifold $(\mathcal{Z}, \omega)$ one can construct a Sasakian structure on the total space of the circle bundle defined by the integral class $[\omega]$ in $H_{\text {orb }}^{2}(\mathcal{Z}, \mathbb{Z})$. 


\section{Deformation Classes of Sasakian Structures}

Sasakian structures like Kähler structures are plentiful; in fact, the 'moduli space' is infinite dimensional. It is thus important to look for distinguished Sasakian metrics in a given deformation class, and $\eta$-Einstein metrics provide good examples. There are two essential types of deformations of Sasakian structures, those that deform the characteristic foliation (deformations of type $I$ ) and those that fix the characteristic foliation but deform the contact 1-form (deformations of type II).

\section{Deformations of Type I}

Let us consider deformations of a Sasakian structure with a fixed transverse holomorphic structure, that is deformations that deform the characteristic foliation $\mathcal{F}_{\xi}$ by deforming the vector field $\xi$. This type of deformation was first considered by Takahashi Tak78] who considered deformations by adding an infinitesimal automorphism $\rho \in \mathfrak{a} \mathfrak{u t}(\xi, \eta, \Phi, g)$. However, here we consider the more general deformations introduced in G098, and used in the 3-dimensional case by Belgun Bel01. Explicitly, we consider deformations of the contact form whose Reeb vector field is an infinitesimal automorphism of the CR structure, that is, we consider a positive function $f$ and tensor fields

$$
\tilde{\eta}=f \eta, \quad \tilde{\xi}=\xi+\rho, \quad \tilde{\Phi}=\Phi-\Phi \tilde{\xi} \otimes \tilde{\eta}
$$

such that

$$
\tilde{\eta}(\tilde{\xi})=1, \quad \tilde{\xi}\rfloor d \tilde{\eta}=0, \quad £_{\tilde{\xi}} \tilde{\Phi}=0 .
$$

It is clear from Equations 11 that $\tilde{\eta}$ is a contact 1-form, the contact subbundle $\mathcal{D}=\operatorname{ker} \eta=\operatorname{ker} \tilde{\eta}$ remains unchanged, and that $\tilde{\Phi} \tilde{\xi}=0$. Furthermore, if $X$ is a section of $\mathcal{D}$, then

$$
\tilde{\Phi}^{2} X=\tilde{\Phi}(\Phi X-\tilde{\eta}(X) \Phi \varrho)=\Phi(\Phi X-\tilde{\eta}(\Phi X) \Phi \varrho)=\Phi^{2} X=-X .
$$

Thus, $\tilde{\Phi}^{2}=-\mathbb{I}+\tilde{\xi} \otimes \tilde{\eta}$, so $(\tilde{\xi}, \tilde{\eta}, \tilde{\Phi})$ is an underlying almost contact structure of the contact structure. Notice that $f$ and $\rho$ are related by

$$
f=\frac{1}{1+\eta(\rho)} \text {. }
$$

Next define the Riemannian metric $\tilde{g}$ by

$$
\tilde{g}=d \tilde{\eta} \circ(\tilde{\Phi} \otimes \mathbb{I}) \oplus \tilde{\eta} \otimes \tilde{\eta} .
$$

This metric is compatible with the contact structure and $\tilde{\xi}$ is a Killing vector field of $\tilde{g}$. Thus, $(\tilde{\xi}, \tilde{\eta}, \tilde{\Phi}, \tilde{g})$ is K-contact. In fact, we claim that it is Sasakian. To see this we need to check normality. But according to Proposition 3.2 of BG01a the structure $(\tilde{\xi}, \tilde{\eta}, \tilde{\Phi}, \tilde{g})$ is normal if and only if the almost CR-structure $\left.\tilde{\Phi}\right|_{\mathcal{D}}$ is integrable and $\tilde{\Phi}$ is invariant under $\tilde{\xi}$. The latter conditions both hold by Equations [1] and [12] We have arrived at

Theorem 4. Let $(\xi, \eta, \Phi, g)$ be a Sasakian structure on $M$. Then the deformations of type I are deformations through Sasakian structures.

The metrics $\tilde{g}$ and $g$ are related by

$$
\tilde{g}=\frac{g-\tilde{\eta} \otimes \tilde{\xi}\rfloor g-\tilde{\xi}\rfloor g \otimes \tilde{\eta}+g(\tilde{\xi}, \tilde{\xi}) \tilde{\eta} \otimes \tilde{\eta}}{1+\eta(\rho))}+\tilde{\eta} \otimes \tilde{\eta}
$$


A very special case of type I deformations occur when $\rho=c \xi$ for some constant $c$. This does not deform the characteristic foliation, but only rescales the vector field $\xi$ and contact 1 -form $\eta$. It is convenient to write this transformation in the form

$$
\xi^{\prime}=a^{-1} \xi, \quad \eta^{\prime}=a \eta, \quad \Phi^{\prime}=\Phi, \quad g=a g+a(a-1) \eta \otimes \eta,
$$

where $a$ is constant. These were called $\mathcal{D}$-homothetic deformations by Tanno Tan68. We have also referred to them as transverse homotheties.

\section{Deformations of Type II}

Now fix a Sasakian structure $(\xi, \eta, \Phi, g)$ on $M^{2 n+1}$ and consider deformations which stabilize the characteristic foliation $\mathcal{F}_{\xi}$. More explicitly we wish to deform $(\xi, \eta, \Phi, g)$ through Sasakian structures that have the same fundamental basic cohomology class up to a scale. As in Kähler geometry this deformation space is infinite dimensional. We consider a deformation of the structure $(\xi, \eta, \Phi, g)$ by adding to $\eta$ a continuous one parameter family of 1 -forms $\zeta_{t}$ that are basic with respect to the characteristic foliation. We require that the 1 -form $\eta_{t}=\eta+\zeta_{t}$ satisfy the conditions

$$
\eta_{0}=\eta, \quad \zeta_{0}=0, \quad \eta_{t} \wedge\left(d \eta_{t}\right)^{n} \neq 0 \quad \forall t \in[0,1] .
$$

This last non-degeneracy condition implies that $\eta_{t}$ is a contact form on $M$ for all $t \in[0,1]$ which by Gray's Stability Theorem Gra59 belongs to the same underlying contact structure as $\eta$. Moreover, since $\zeta_{t}$ is basic $\xi$ is the Reeb (characteristic) vector field associated to $\eta_{t}$ for all $t$. Now let us define

$$
\begin{aligned}
\Phi_{t} & =\Phi-\xi \otimes \zeta_{t} \circ \Phi \\
g_{t} & =d \eta_{t} \circ\left(\Phi \otimes \mathbb{I}_{t}\right)+\eta_{t} \otimes \eta_{t} .
\end{aligned}
$$

In BG03] it was proved that for all $t \in[0,1]$ and every basic 1 -form $\zeta_{t}$ such that $d \zeta_{t}$ is of type $(1,1)$ and such that (13) holds $\left(\xi, \eta_{t}, \Phi_{t}, g_{t}\right)$ defines a continuous 1parameter family of Sasakian structures on $M$ belonging to the same underlying contact structure as $\eta$.

Theorem 5. Let $(M, \xi, \eta, \Phi, g)$ be a Sasakian manifold. Then for all $t \in[0,1]$ and every basic 1-form $\zeta_{t}$ such that $d \zeta_{t}$ is of type $(1,1)$ and such that 13) holds $\left(\xi, \eta_{t}, \Phi_{t}, g_{t}\right)$ defines a Sasakian structure on $M$ belonging to the same underlying contact structure as $\eta$.

Given a Sasakian structure $\mathcal{S}=(\xi, \eta, \Phi, g)$ on a manifold $M$, we defined $\mathfrak{F}(\xi)$ BGN03b to be the family of all Sasakian structures obtained by deformations of type II. Notice that any two Sasakian structures $(\xi, \eta, \Phi, g)$ and $\left(\xi^{\prime}, \eta^{\prime}, \Phi^{\prime}, g^{\prime}\right)$ in $\mathfrak{F}(\xi)$ are homologous in the sense that $\left[d \eta^{\prime}\right]_{B}=[d \eta]_{B}$. This definition was then extended to include transverse homotheties in BGN03c. We defined $\mathfrak{F}\left(\mathcal{F}_{\xi}\right)$ to be the set of all Sasakian structures whose characteristic foliation is $\mathcal{F}_{\xi}$. Clearly, we have $\mathfrak{F}(\xi) \subset \mathfrak{F}\left(\mathcal{F}_{\xi}\right)$. For any Sasakian structure $\mathcal{S}=(\xi, \eta, \Phi, g)$ there is the "conjugate Sasakian structure" defined by $\mathcal{S}^{c}=\left(\xi^{c}, \eta^{c}, \Phi^{c}, g\right)=(-\xi,-\eta,-\Phi, g) \in \mathfrak{F}\left(\mathcal{F}_{\xi}\right)$. So fixing $\mathcal{S}$ we define

$$
\mathfrak{F}^{+}\left(\mathcal{F}_{\xi}\right)=\bigcup_{a \in \mathbb{R}^{+}} \mathfrak{F}\left(a^{-1} \xi\right)
$$

and $\mathfrak{F}^{-}\left(\mathcal{F}_{\xi}\right)$ to be the image of $\mathfrak{F}^{+}\left(\mathcal{F}_{\xi}\right)$ under conjugation. It is then easy to see that we have a decomposition $\mathfrak{F}\left(\mathcal{F}_{\xi}\right)=\mathfrak{F}^{+}\left(\mathcal{F}_{\xi}\right) \sqcup \mathfrak{F}^{-}\left(\mathcal{F}_{\xi}\right)$. Two Sasakian structures 
$\mathcal{S}=(\xi, \eta, \Phi, g)$ and $\mathcal{S}^{\prime}=\left(\xi^{\prime}, \eta^{\prime}, \Phi^{\prime}, g^{\prime}\right)$ on a smooth manifold $M$ are said to be a-homologous if there is an $a \in \mathbb{R}^{+}$such that $\xi^{\prime}=a^{-1} \xi$ and $\left[d \eta^{\prime}\right]_{B}=a[d \eta]_{B}$.

More generally it is convenient to include both deformations of type I and II as well as deformations of the CR-structure. For a fixed Sasakian structure $\mathcal{S}=$ $(\xi, \eta, \Phi, g)$ we denote by $\mathfrak{F}(\mathcal{S})$ the space of all Sasakian structures that can be obtained from $\mathcal{S}$ by deformations through CR-structures that are compatible with the contact structure or deformations of type I and II above. The topology of $\mathfrak{F}(\mathcal{S})$ is compact open $C^{\infty}$-topology induced by the corresponding tensor fields. The underlying contact structure remains fixed in $\mathfrak{F}(\mathcal{S})$.

\section{4. $\eta$-Einstein Metrics and the Calabi Problem}

In this section we begin the main objects of study, namely $\eta$-Einstein metrics which were introduced and studied by Okumura Oku62 in 1962. In particular, he studied the relation between the existence of $\eta$-Einstein metrics and certain harmonic forms, although the correct cohomological setting and connection to the Calabi problem was not realized until much later.

Definition 6. A Sasakian structure $\mathcal{S}=(\xi, \eta, \Phi, g)$ on $M$ is said to be Sasakian $\eta$-Einstein or just $\eta$-Einstein if there are constants $\lambda, \nu$ such that $\operatorname{Ric}_{g}=\lambda g+\nu \eta \otimes \eta$.

One can obviously generalize this definition to any almost contact metric manifold $(M, \eta, \xi, \Phi, g)$. In such generality one typically asks for $\lambda, \nu \in C^{\infty}(M)$ to be arbitrary functions. However, on a Sasakian or even only K-contact manifold the Ricci curvature tensor satisfies $\operatorname{Ric}_{g}(\xi, X)=2 n \eta(X)$ and it easily follows Oku62. that

Lemma 7. Let $M$ be a $K$-contact manifold of dimension $2 n+1$ such that

$$
\operatorname{Ric}_{g}=\lambda g+\nu \eta \otimes \eta
$$

for some $\lambda, \nu \in C^{\infty}(M)$. Then if $n>1, \lambda$, and $\nu$ are constants satisfying $\lambda+\nu=2 n$.

Remark 8. In the 3-dimensional case any K-contact manifold is automatically Sasakian. A Sasakian 3-manifold $M$ is always $\eta$-Einstein in the wider sense of the definition which allows $\lambda, \nu$ to be functions on $M$. Hence, in this paper we define a Sasakian 3-manifold to be $\eta$-Einstein as in Definition 6 On the other hand, when $M$ is a metric contact manifold one can study metrics for which $\operatorname{Ric}_{g}=\lambda g+\nu \eta \otimes \eta$, with $\lambda, \nu \in C^{\infty}(M)$ (see Bla02]).

An immediate consequence of the definition is

Corollary 9. Every Sasakian $\eta$-Einstein manifold is of constant scalar curvature $s=2 n(\lambda+1)$.

We briefly recall the basic first Chern class from BGN03b. Now the contact subbundle $\mathcal{D}$ is a complex vector bundle and thus has a first Chern class $c_{1}(\mathcal{D}) \in H^{2}(M, \mathbb{Z})$. Consider the long exact sequence 6 together with the natural map $H^{2}(M, \mathbb{Z}) \longrightarrow H^{2}(M, \mathbb{R})$ whose kernel is the torsion part of $H^{2}(M, \mathbb{Z})$. From (5) of Theorem 1 we have

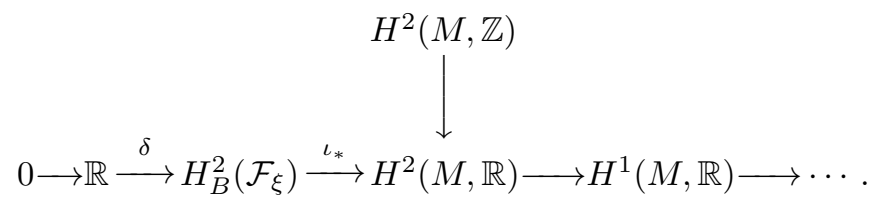


As in (6) the map $\delta$ is given by $\delta(c)=c[d \eta]$ where $c \in \mathbb{R}$. Now on a Sasakian manifold the vector bundle $\mathcal{D}^{1,0}$ is holomorphic with respect to the CR-structure, so we can compute the free part of $c_{1}(\mathcal{D})=c_{1}\left(\mathcal{D}^{1,0}\right)$ from the transverse Kähler geometry in the usual way. That is $c_{1}(\mathcal{D})$ can be represented by a basic real closed $(1,1)$-form $\rho_{B}$. The class $c_{1}^{B}=\left[\rho_{B}\right] \in H_{B}^{2}\left(\mathcal{F}_{\xi}\right)$ is independent of the transverse metric and basic connection used to compute it, and depends only on the foliated manifold $\left(M, \mathcal{F}_{\xi}\right)$ with its CR-structure. It is described in EKA90 and called the basic first Chern class of $\mathcal{D}$ there.

Definition 10. A Sasakian structure $(\xi, \eta, \Phi, g)$ is said to be positive (negative) if $c_{1}^{B}$ is represented by a positive (negative) definite $(1,1)$-form. If either of these two conditions is satisfied $(\xi, \eta, \Phi, g)$ is said to be definite, and otherwise $(\xi, \eta, \Phi, g)$ is called indefinite. $(\xi, \eta, \Phi, g)$ is said to be null if $c_{1}^{B}=0$.

In analogy with common terminology of smooth algebraic varieties we see that a positive Sasakian structure is a transverse Fano structure, while a null Sasakian structure is a transverse Calabi-Yau structure. The negative Sasakian case corresponds to the canonical bundle being ample; we refer to this as a transverse canonical structure.

Recall the transverse Ricci tensor $\operatorname{Ric}_{g}^{T}$ of $g_{T}$ to be the Ricci tensor of the transverse metric $g_{T}$. It is related to the Ricci tensor $\operatorname{Ric}_{g}$ of $g$

$$
\operatorname{Ric}(X, Y)=\operatorname{Ric}_{T}(X, Y)-2 g(X, Y) .
$$

Now as usual define the Ricci form $\rho_{g}$ and transverse Ricci form $\rho_{g}^{T}$ by

$$
\rho_{g}(X, Y)=\operatorname{Ric}_{g}(X, \Phi Y), \quad \rho_{g}^{T}(X, Y)=\operatorname{Ric}_{g}^{T}(X, \Phi Y)
$$

for smooth sections $X, Y$ of $\mathcal{D}$. It is easy to check that these are anti-symmetric of type $(1,1)$ and 17 implies that they are related by

$$
\rho_{g}^{T}=\rho_{g}+2 d \eta
$$

Thus, as in the usual case we have

Lemma 11. The basic class $2 \pi c_{1}^{B} \in H_{B}^{1,1}\left(\mathcal{F}_{\xi}\right)$ is represented by the transverse Ricci form $\rho_{g}^{T}$.

In BGN03b] we used El Kacimi-Alaoui's 'Transverse Yau Theorem' EKA90 to prove the following converse to Lemma 11]

Theorem 12. Let $(M, \xi, \eta, \Phi, g)$ be a Sasakian manifold whose basic first Chern class $c_{1}^{B}$ is represented by the real basic $(1,1)$ form $\rho$, then there is a unique Sasakian structure $\left(\xi, \eta_{1}, \Phi_{1}, g_{1}\right) \in \mathfrak{F}(\xi)$ homologous to $(\xi, \eta, \Phi, g)$ such that $\rho_{g_{1}}=\rho-2 d \eta_{1}$ is the Ricci form of $g_{1}$.

In Problem 16] below we give another version of the Sasakian Calabi Problem. We are interested in certain invariance properties of $c_{1}^{B}$. Since the transverse Ricci form $\rho_{g}^{T}$ is invariant under scaling, we see that $c_{1}^{B}$ is independent of the Sasakian structure in $\mathfrak{F}\left(\mathcal{F}_{\xi}\right)$. Now from the exact sequence (16) we have

Proposition 13. Let $(\xi, \eta, \Phi, g)$ be a Sasakian structure with underlying contact bundle $\mathcal{D}$. Then $c_{1}(\mathcal{D})$ is a torsion class if and only if there exists a real number a such that $c_{1}^{B}=a[d \eta]_{B}$. In particular,

(1) If $c_{1}(\mathcal{D})$ is a torsion class then every Sasakian structure in $\mathfrak{F}(\mathcal{S})$ is either definite or null. 
(2) If $\mathfrak{F}\left(\mathcal{F}_{\xi}\right)$ admits a Sasakian $\eta$-Einstein structure, then $c_{1}(\mathcal{D})$ is a torsion class.

Hence, a non-torsion $c_{1}(\mathcal{D})$ is the obstruction to the existence of a Sasakian $\eta$-Einstein metric. In particular, in the case when $\lambda>-2$ there is a canonical variation to a Sasakian-Einstein metric [Tan79], so $c_{1}(\mathcal{D})$ is an obstruction to the existence of a Sasakian-Einstein metric on $M$ in the given $\xi$-deformation class BG01a. Since $c_{1}(\mathcal{D})$ is an invariant of the complex vector bundle $\mathcal{D},(2)$ gives an obstruction to the type of contact structure that can admit a compatible Sasakian $\eta$-Einstein metric. However, since $c_{1}(\mathcal{D})$ is related to a topological invariant, namely the second Stiefel-Whitney class $w_{2}(M)$, there are topological obstructions to the existence of Sasakian $\eta$-Einstein metrics. From the natural splitting $T M=\mathcal{D} \oplus L_{\xi}$ where $L_{\xi}$ is the trivial real line bundle generated by $\xi$, one gets

$$
w_{2}(M)=w_{2}(T M)=w_{2}(\mathcal{D})
$$

which is the mod 2 reduction of $c_{1}(\mathcal{D}) \in H^{2}(M, \mathbb{Z})$. Since a manifold $M$ admits a spin structure if and only if $w_{2}(M)=0$, Proposition 13 implies

Theorem 14. Let $M$ be a non-spin manifold (i.e., $w_{2}(M) \neq 0$ ) with $H_{1}(M, \mathbb{Z})$ torsion free. Then $M$ does not admit a Sasakian $\eta$-Einstein structure.

Thus, Theorem 14 includes as a special case the well-known fact BG00, Mor97 that a simply connected Sasakian-Einstein manifold is necessarily spin. Next we give an example of a Sasakian manifold that does not admit a Sasakian $\eta$-Einstein metric.

Example 15. Consider the non-trivial $S^{3}$-bundle over $S^{2}$, denoted by $X_{\infty}$ in Barden Bar65. We represent $X_{\infty}$ as a non-trivial circle bundle over a Hirzebruch surface. Recall the Hirzebruch surfaces $S_{n}$ [GH78] are realized as the projectivizations of the sum of two line bundles over $\mathbb{C P}^{1}$, namely

$$
S_{n}=\mathbb{P}(\mathcal{O} \oplus \mathcal{O}(-n)) .
$$

They are diffeomorphic to $\mathbb{C P}^{1} \times \mathbb{C P}^{1}$ if $n$ is even, and to the blow-up of $\mathbb{C P}^{2}$ at one point, which we denote as $\widetilde{\mathbb{C P}}^{2}$, if $n$ is odd. We need to take $n$ odd to get a non-spin circle bundle. Now $\operatorname{Pic}\left(S_{n}\right) \approx \mathbb{Z} \oplus \mathbb{Z}$, and we can take the Poincaré duals of a section of $\mathcal{O}(-n)$ and the homology class of the fiber as its generators. The corresponding divisors can be represented by rational curves which we denote by $C$ and $F$, respectively satisfying

$$
C \cdot C=-n, \quad F \cdot F=0, \quad C \cdot F=1 .
$$

Let $\alpha$ and $\beta$ denote the Poincaré duals of $C$ and $F$, respectively, and write the Kähler form $\omega$ so that $[\omega]=a \alpha+b \beta$. According to HS97 every Kähler class can be represented in this form with $b=2$ and $a>0$. Let

$$
S^{1} \longrightarrow M \stackrel{\pi}{\longrightarrow} S_{n}
$$

be the circle bundle over $S_{n}$ whose first Chern class is [ $\left.\omega\right]$. Since we want $M$ simply connected, we need to choose $\operatorname{gcd}(a, b)=1$. It suffices to take $n=3$, and $[\omega]=$ $\alpha+2 \beta$. Now $c_{1}(\mathcal{D})=\pi^{*} c_{1}\left(S_{n}\right)=-\pi^{*} c_{1}(K)$ where $K$ is the canonical divisor of $S_{n}$. From GH78, we have $K=-2 C-(n+2) F$. So in our case we have $c_{1}(K)=-2 \alpha-5 \beta$. From the Gysin sequence of the circle bundle we have

$$
0 \longrightarrow \mathbb{Z} \stackrel{\cup[\omega]}{\longrightarrow} H^{2}\left(S_{n}, \mathbb{Z}\right) \stackrel{\pi^{*}}{\longrightarrow} H^{2}(M, \mathbb{Z}) \longrightarrow 0,
$$


giving $c_{1}(\mathcal{D})=\pi^{*}\left(c_{1}(-K)\right)=\pi^{*}(2 \alpha+5 \beta)=\pi^{*} \beta$. Hence, $w_{2}(M) \neq 0$ implying that $M=X_{\infty}$ by Barden's Classification Theorem Bar65]. Thus, we have produced a Sasakian structure on $X_{\infty}$, but by Theorem 14 it cannot admit a Sasakian $\eta$ Einstein structure.

We are interested in proving the existence of Sasakian $\eta$-Einstein metrics in a given deformation class of Sasakian structures.

Problem 16. [Sasakian Calabi Problem] Given a manifold $M$ with Sasakian structure $\mathcal{S}=(\xi, \eta, \Phi, g)$ and with basic first Chern class $c_{1}^{B}$ that is represented by either a positive definite, negative definite real basic $(1,1)$ form $\rho^{T}$, or if $c_{1}^{B}$ vanishes, does there exist a Sasakian structure $\mathcal{S}^{\prime} \in \mathfrak{F}\left(\mathcal{F}_{\xi}\right)$ with an $\eta$-Einstein metric $g^{\prime}$ ?

The solution to this problem is, of course, given in local CR-coordinates $\left(z_{i}, \bar{z}_{i}, x\right)$ on $M$ by the "transverse Monge-Ampère equation"

$$
\frac{\operatorname{det}\left(g_{i \bar{j}}^{T}+\phi_{i \bar{j}}\right)}{\operatorname{det}\left(g_{i \bar{j}}^{T}\right)}=e^{-k \phi+F}, \quad g_{i \bar{j}}^{T}+\phi_{i \bar{j}}>0
$$

where $g^{T}$ is the transverse metric, $\phi$ and $F$ are real basic functions, and $\phi_{i \bar{j}}$ are the components of $\partial \bar{\partial} \phi=d \zeta_{t}$ with respect to the transverse coordinates $\left(z_{i}, \bar{z}_{\bar{j}}\right)$. The cases when $c_{1}^{B}$ is zero, positive or negative definite correspond to finding solutions of equation 20] with the constant $k=0,>0,<0$, respectively. In the negative and zero cases we have the transverse version of theorems of Yau Yau78 and Aubin Aub82 give

Theorem 17. If the class $\left[\rho^{T}\right] \in H_{B}^{2}\left(\mathcal{F}_{\xi}\right)$ is zero or can be represented by a negative definite $(1,1)$ form, then there exists a Sasakian structure $\mathcal{S} \in \mathfrak{F}(\xi)$ with an $\eta$ Einstein metric $g$ on $M$ with $\lambda=-2$ in the first case and $\lambda<-2$ in the second.

In other words, in complete analogy with the Kählerian case, we see that there are no obstructions to solving Sasakian or transverse Monge-Ampère problem in the negative or null case.

\section{Further Results on $\eta$-Einstein Metrics}

Let us now consider a Sasakian $\eta$-Einstein with Sasakian structure $\mathcal{S}=(\xi, \eta, \Phi, g)$ and $\eta$-Einstein constants $(\lambda, \nu)$. A simple calculation shows that $\mathcal{D}$-homotheties preserve $\eta$-Einstein condition. More precisely, we have

Proposition 18. Let $(M, \xi, \eta, \Phi, g)$ be a Sasakian $\eta$-Einstein manifold with constants $(\lambda, \nu)$. Consider a D -homothetic Sasakian structure $\mathcal{S}^{\prime}=\left(\xi^{\prime}, \eta^{\prime}, \Phi^{\prime}, g^{\prime}\right)=$ $\left(a^{-1} \xi, a \eta, \Phi, a g+a(a-1) \eta \otimes \eta\right)$. Then $\left(M, \mathcal{S}^{\prime}\right)$ is also $\eta$-Einstein with constants

$$
\lambda^{\prime}=\frac{\lambda+2-2 a}{a}, \quad \nu^{\prime}=2 n-\frac{\lambda+2-2 a}{a} .
$$

Proof. The proof follows from a simple formula relating Ricci curvature tensors of the Sasakian metric $g^{\prime}, g$ related by a $\mathcal{D}$-homothetic transformation [Tan68]:

$$
\operatorname{Ric}_{g^{\prime}}=\operatorname{Ric}_{g}-2(a-1) g+(a-1)(2 n+2+2 n a) \eta \otimes \eta .
$$


Note that $\mathcal{D}$-homotheties must preserve the sign of the Sasakian $\eta$-Einstein structure (invariance of basic Chern classes) and they are completely analogous to the usual homotheties of Einstein metrics. When $(M, \xi, \eta, \Phi, g)$ is null then always $(\lambda, \nu)=(-2,2 n+2)$. When $(M, \xi, \eta, \Phi, g)$ is positive then $\lambda>-2$. But by applying a suitable $\mathcal{D}$-homothety one can get any value of $\lambda \in(-2, \infty)$. Similarly, when $(M, \xi, \eta, \Phi, g)$ is negative $\lambda<-2$ and by applying a suitable $\mathcal{D}$-homothety one can get any value of $\lambda \in(-\infty,-2)$.

An easy computation shows that, in addition to the structure Theorem 3 in the $\eta$-Einstein case we can actually say more.

Theorem 19. Let $(M, \xi, \eta, \Phi, g)$ be a compact quasi-regular Sasakian $\eta$-Einstein manifold of dimension $2 n+1$, and let $\mathcal{Z}$ denote the space of leaves of the characteristic foliation. Then the leaf space $\mathcal{Z}$ is a Hodge orbifold with Kähler-Einstein metric $h$ with Einstein constant $\lambda+2$.

The case of $\lambda+2>0$ is special as follows from Proposition 18. By applying suitable $\mathcal{D}$-homothety one can always choose $\lambda=2 n$ which turns the $\eta$-Einstein metric into an Einstein metric. This observation is originally due to Tanno who used it to show that a unit tangent bundle of $S^{n}$ has a homogeneous SasakianEinstein structure. When $n=3$ one gets a homogeneous Sasakian-Einstein metric on $S^{2} \times S^{3}$ Tan79. We have

Theorem 20. Let $(M, \xi, \eta, \Phi, g)$ be a compact quasi-regular Sasakian $\eta$-Einstein manifold of dimension $2 n+1$, and let $\lambda>-2$. Let $(\mathcal{Z}, h)$ denote the space of leaves of the characteristic foliation with its Kähler-Einstein metric $h$. Then $M$ admits a Sasakian-Einstein structure $\left(\xi^{\prime}, \eta^{\prime}, \Phi^{\prime}, g^{\prime}\right) \in \mathfrak{F}\left(\mathcal{F}_{\xi}\right)$, D-homothetic to $\mathcal{S}$, with $g^{\prime}=\alpha g+\alpha(\alpha-1) \eta \otimes \eta$ and $\alpha=\frac{\lambda+2}{2 n+2}$.

It is useful therefore to think of positive Sasakian $\eta$-Einstein structure as a family parameterized by a positive constant $a \in \mathbb{R}^{+}$. In addition, if we choose the Sasakian-Einstein structure in this family $\mathcal{S}_{1}$ as a reference point we will distinguished between two subfamilies.

Definition 21. Let $\left(M, \xi_{1}, \eta_{1}, \Phi_{1}, g_{1}\right)$ be a compact quasi-regular Sasakian-Einstein manifold of dimension $2 n+1$, and let $\mathcal{S}_{a}=\left(\xi_{a}, \eta_{a}, \Phi_{a}, g_{a}\right)$ be the Sasakian $\eta$-Einstein structure obtained from $\mathcal{S}_{1}$ by applying $\mathcal{D}$-homothetic transformation to $\mathcal{S}_{1}$ with constant $a \in \mathbb{R}^{+}$. We say that $\mathcal{S}_{a}$ is stretched when $a>1(-2<\lambda<2 n, \nu>0)$ and squashed when $0<a<1(\lambda>2 n, \nu<0)$.

Note that positive squashed Sasakian $\eta$-Einstein manifolds can have scalar curvature of any sign. There is a unique metric in the family $\mathcal{S}_{a}$ which makes $\lambda=-1$ in which case the Sasakian manifold becomes scalar-flat.

In the negative case there is another interesting structure related to Sasakian $\eta$-Einstein metric. Recall Bau00, Boh03] the following

Definition 22. Let $(M, g)$ be a Lorentzian manifold of dimension $2 n+1$ and let $\xi$ be a time-like Killing vector field such that $g(\xi, \xi)=-1$. We say that that $M$ is Sasakian if $\Phi(X)=\nabla_{X} \xi$ satisfies the condition $\left(\nabla_{X} \Phi\right)(Y)=g(X, \xi) Y+g(X, Y) \xi$ and Sasakian-Einstein if, in addition, $g$ is Einstein.

Equivalently, we can require the metric cone $\mathcal{C}(M)=\left(\mathbb{R}^{+} \times M,-d t^{2}+r^{2} g, d\left(r^{2} \eta\right)\right)$ to be pseudo-Kähler of signature $(2,2 n)$. In the Sasakian-Einstein case the cone metric is pseudo-Calabi-Yau and the Einstein constant must equal to $-2 n$. This 
is in complete analogy with the Riemannian case. Geometries of this type are interesting as they provide examples of the so-called twistor spinors on Lorentzian manifolds. As a simple consequence we get

Proposition 23. Let $(M, \xi, \eta, \Phi, g)$ be a compact quasi-regular Sasakian $\eta$-Einstein manifold of dimension $2 n+1$, and let $\lambda<-2$. Let $(\mathcal{Z}, h)$ denote the space of leaves of the characteristic foliation with its Kähler-Einstein metric h. Then $M$ admits a Lorentzian Sasakian-Einstein structure such that

$$
\xi^{\prime}=a^{-1} \xi, \quad-g^{\prime}=a g+a(a-1) \eta \otimes \eta,
$$

where $a=\frac{\lambda+2}{2+2 n}$.

Combining this with Theorem [17 we have

Corollary 24. Every negative Sasakian manifold admits a Lorentzian SasakianEinstein structure.

\section{6. $\eta$-Einstein Structures on Links}

Let $\mathbf{w}=\left(w_{0}, \ldots, w_{n}\right)$ be any positive vector in $\mathbb{R}^{n+1}$, that is $w_{i}>0$ for all $i=0, \ldots, n$. The vector field corresponding to $\mathbf{w}$ is denoted by $\xi_{\mathbf{w}}$, and the corresponding 1 -form by $\eta_{\mathbf{w}}$. Let $\left(x_{0}, \ldots, x_{n}, y_{0}, \ldots, y_{n}\right)$ be the coordinates on the unit sphere $S^{2 n+1}$ in $\mathbb{R}^{2 n+2}$. A family Sasakian structures $\mathcal{S}_{\mathbf{w}}=\left(\xi_{\mathbf{w}}, \eta_{\mathbf{w}}, \Phi_{\mathbf{w}}, g_{\mathbf{w}}\right)$ is now defined by

$$
\xi_{\mathbf{w}}=\sum_{i=0}^{n} w_{i}\left(y_{i} \partial_{x_{i}}-x_{i} \partial_{y_{i}}\right), \quad \eta_{\mathbf{w}}=\frac{\sum_{i=0}^{n} x_{i} d y_{i}}{\sum_{i=0}^{n} w_{i}\left(\left(x^{i}\right)^{2}+\left(y^{i}\right)^{2}\right)}
$$

with $\Phi_{\mathbf{w}}$ and $g_{\mathbf{w}}$ further determined by $\xi_{\mathbf{w}}$ and $\eta_{\mathbf{w}}$. We shall refer to $\left(\xi_{\mathbf{w}}, \eta_{\mathbf{w}}, \Phi_{\mathbf{w}}, g_{\mathbf{w}}\right)$ as the weighted Sasakian structure on $S^{2 n+1}$, and we denote the unit sphere with this Sasakian structure by $S_{\mathbf{w}}^{2 n+1}$. Note that the standard Sasakian-Einstein structure (Hopf fibration) corresponds to setting $\mathbf{w}=(1, \ldots, 1)$. The deformed structures $\left(\xi_{\mathbf{w}}, \eta_{\mathbf{w}}, \Phi_{\mathbf{w}}, g_{\mathbf{w}}\right)$ are not all distinct. The Weyl group $\mathcal{W}(S U(n+1))=$ $\operatorname{Nor}\left(\mathfrak{T}_{n+1}\right) / \mathfrak{T}_{n+1}$ acts as outer automorphisms on the maximal torus, and it is well-known that $\mathcal{W}(S U(n+1))=\Sigma_{n+1}$, the permutation group on $n+1$ letters. So for any $\sigma \in \Sigma_{n+1}$ the Sasakian structures $\mathcal{S}_{\mathbf{w}}=\left(\xi_{\mathbf{w}}, \eta_{\mathbf{w}}, \Phi_{\mathbf{w}}, g_{\mathbf{w}}\right)$ and $\left(\xi_{\sigma(\mathbf{w})}, \eta_{\sigma(\mathbf{w})}, \Phi_{\sigma(\mathbf{w})}, g_{\sigma(\mathbf{w})}\right)$ are isomorphic. Thus, we shall frequently take the weights to be ordered such that $w_{0} \leq w_{1} \leq \cdots \leq w_{n}$. It is important to note that all the Sasakian structures $\mathcal{S}_{\mathbf{w}}$ belong to the same underlying contact structure on $S^{2 n+1}$, namely the standard one.

Consider the affine space $\mathbb{C}^{n+1}$ together with a weighted $\mathbb{C}^{*}$-action given by

$$
\left(z_{0}, \ldots, z_{n}\right) \mapsto \lambda \cdot \mathbf{z}=\left(\lambda^{w_{0}} z_{0}, \ldots, \lambda^{w_{n}} z_{n}\right),
$$

where $\lambda \in \mathbb{C}^{*}$, and the weights $w_{j}$ are positive integers.

Definition 25. A polynomial $f \in \mathbb{C}\left[z_{0}, \ldots, z_{n}\right]$ is said to be weighted homogeneous of degree $d$ if there are positive integers $w_{0}, w_{1}, \ldots, w_{n}, d$ such that

$$
f(\lambda \cdot \mathbf{z})=f\left(\lambda^{w_{0}} z_{0}, \ldots, \lambda^{w_{n}} z_{n}\right)=\lambda^{d} f\left(z_{0}, \ldots, z_{n}\right)=\lambda^{d} f(\mathbf{z}) .
$$


We shall assume that $w_{0} \leq w_{1} \leq \cdots \leq w_{n}$ and that $\operatorname{gcd}\left(w_{0}, \ldots, w_{n}\right)=1$ unless otherwise stated. The zero set $V_{f}$ of a non-degenerate weighted homogeneous polynomial $f$, is just the weighted affine cone $C_{f}$. If the origin is an isolated singularity in $C_{f}$, then it is the only singularity of $C_{f}$. We shall assume this from now on. In this case we can take $\epsilon=1$ so that $S_{\epsilon}^{2 n+1}\left(\mathbf{z}_{0}\right)$ becomes the unit sphere $S^{2 n+1}$ centered at the origin with its weighted Sasakian structure $\mathcal{S}_{\mathbf{w}}$. In this case our link

$$
L_{f}=C_{f} \cap S^{2 n+1} .
$$

is a smooth manifold of dimension $2 n-1$ which by the Milnor Fibration Theorem is $(n-2)$-connected.

One can easily see that every link is either positive, negative, or null. More precisely, we have BGK03

Theorem 26. Let $f$ be a non-degenerate weighted homogeneous polynomial of degree $d$ and weight vector $\mathbf{w}$. The Sasakian structure $\left(\xi_{\mathbf{w}}, \eta_{\mathbf{w}}, \Phi_{\mathbf{w}}, g_{\mathbf{w}}\right)$ on $S^{2 n+1}$ induces by restriction a Sasakian structure, denoted by $\mathcal{S}_{\mathbf{w}, f}=\left.\mathcal{S}_{\mathbf{w}}\right|_{L_{f}}$ on the link $L_{f}$. Furthermore, let $|\mathbf{w}|=w_{0}+\cdots+w_{n}$. Then

(1) $\mathcal{S}_{\mathbf{w}, f}$ is positive when $d-|\mathbf{w}|<0$,

(2) $\mathcal{S}_{\mathbf{w}, f}$ is null when $d-|\mathbf{w}|=0$,

(3) $\mathcal{S}_{\mathbf{w}, f}$ is negative when $d-|\mathbf{w}|>0$.

Example 27. Let $f(\mathbf{z})=z_{0}^{a_{0}}+\cdots+z_{n}^{a_{n}}$. Then the link $L_{f}$ is called the BrieskornPham link and we will denote it by $L(\mathbf{a})=L\left(a_{0}, \ldots, a_{n}\right)$. It can be seen that the weighted degree of the Brieskorn-Pham polynomial is $d=\operatorname{lcm}\left(a_{0}, \ldots, a_{n}\right)$ and the weights are $w_{j}=d / a_{j}$. Then by Theorem 26

(1) $L(\mathbf{a})$ is positive when $\sum_{i} \frac{1}{a_{i}}>1$,

(2) $L(\mathbf{a})$ is null when $\sum_{i} \frac{1}{a_{i}}=1$,

(3) $L(\mathbf{a})$ is negative when $\sum_{i} \frac{1}{a_{i}}<1$.

Theorem 26 together with Theorem 17provide a rich source of $\eta$-Einstein metrics in the non-positive case where there are no obstructions. Summarizing we have

Theorem 28. Let $\mathcal{S}_{\mathrm{w}, f}$ be null or negative. Then $L_{f}$ admits a Sasakian structure $\mathcal{S}^{\prime}$ with $\eta$-Einstein metric $g^{\prime}$ in the same deformation class as $\mathcal{S}_{\mathbf{w}, f}$.

In the Fano case there are obstructions to the existence of such structures. However, as every $\eta$-Sasakian metric can be deformed to a Sasakian-Einstein one such obstruction are completely equivalent to the obstructions preventing existence of orbifold Kähler-Einstein metric on the transverse space $\mathcal{Z}_{f}$. Existence of KählerEinstein metrics on various Fano orbifolds can be often established by the continuity method (cf. BGK03 and references therein).

We finish this section by reviewing very briefly how one determines the topology of the links. The procedure is that of Milnor and Orlik MO70] and involves computing the Alexander polynomial of the link. For more details we refer to MO70 and our previous work BG01b, BGN03b. Milnor and Orlik give a combinatorial formula for the $(n-1)$-st Betti number of a $(2 n-1)$-dimensional link, viz.

$$
b_{n-1}\left(L_{f}\right)=\sum(-1)^{n+1-s} \frac{u_{i_{1}} \cdots u_{i_{s}}}{v_{i_{1}} \cdots v_{i_{s}} \operatorname{lcm}\left(u_{i_{1}}, \ldots, u_{i_{s}}\right)},
$$


where the quotients $d / w_{i}$ are written in the irreducible form $u_{i} / v_{i}$, and the sum is taken over all the $2^{n+1}$ subsets $\left\{i_{1}, \ldots, i_{s}\right\}$ of $\{0, \ldots, n\}$. In the case of determining homology spheres or more generally rational homology spheres it is more efficient to use the Brieskorn Graph Theorem. We refer to BGK03 for details. Another important result proven in BG01b concerns 5-dimensional manifolds only. It says that if the weights $\left(w_{0}, w_{1}, w_{2}, w_{3}\right)$ satisfy the "well-formedness" condition $\operatorname{gcd}\left(w_{i}, w_{j}, w_{k}\right)=1$ for all distinct $i, j, k$, then the 5-dimensional link $L_{f}$ has no torsion.

\section{Sasakian Structures in Dimension 3}

Three dimensional Sasakian geometry is well understood, culminating in the recent uniformization theorem due to Belgun Bel00 which we state precisely below. Geiges Gei97 showed that a compact 3-manifold admits a Sasakian structure if and only if it is diffeomorphic to one of the following:

(1) $S^{3} / \Gamma$ with $\Gamma \subset \mathfrak{I}_{0}\left(S^{3}\right)=S O(4)$.

(2) $\widetilde{S L}(2, \mathbb{R}) / \Gamma$ where is universal cover of $S L(2, \mathbb{R})$ and $\Gamma \subset \mathfrak{I}_{0}(\widetilde{S L}(2, \mathbb{R}))$.

(3) $\mathrm{Nil}^{3} / \Gamma$ with $\Gamma \subset \mathfrak{I}_{0}\left(\mathrm{Nil}^{3}\right)$.

Here $\Gamma$ is a discrete subgroup of the connected component $\mathfrak{I}_{0}$ of the corresponding isometry group with respect to a 'natural metric', and $\mathrm{Nil}^{3}$ denotes the 3 by 3 nilpotent real matrices, otherwise known as the Heisenberg group. These are three of the eight model geometries of Thurston [Thu97, and correspond precisely to the compact Seifert bundles with non-zero Euler characteristic Sco83. For further discussion of the isometry groups we refer to Sco83. We refer to the three model geometries above as spherical, $\widetilde{S L_{2}}$ type, and nil geometry, respectively.

The purpose of this section then is to describe some examples represented as links of isolated hypersurface singularities. First note that in dimension 3 the basic cohomology group $H^{2}\left(\mathcal{F}_{\xi}\right)$ is 1-dimensional so we cannot get indefinite forms other than zero. Hence

Proposition 29. Let $M$ be a compact 3-dimensional Sasakian manifold. Then $M$ is is either positive, negative, or null.

These 3 types of Sasakian structures correspond precisely to the 3 model geometries above. Belgun's Theorem uniformizes these three cases. We prefer to rephrase Belgun's theorem in terms of the earlier work of Tanno Tan69 on constant $\Phi$ sectional curvature. In dimension three the $\Phi$-sectional curvature is determined by one function, namely $H(X)=K(X, \Phi X)$. Thus, in analogy with 3-dimensional Einstein geometry we have (see also Gui02])

Proposition 30. A three dimensional Sasakian manifold is $\eta$-Einstein if and only if it has constant $\Phi$-sectional curvature.

Proof. The if part, which holds in all dimensions, follows easily from Tanno's classification theorem Tan69. To prove the only if part we choose a local orthonormal bases $X, \Phi X, \xi$ for the Sasakian structure $\mathcal{S}=(\xi, \eta, \Phi, g)$. Now in three dimensions the Ricci curvature and the sectional curvature are related by Pet98.

$$
\begin{aligned}
\operatorname{Ric}_{g}(X, X) & =K(X, \Phi X)+K(X, \xi)=K(X, \Phi X)+1 \\
\operatorname{Ric}_{g}(\Phi X, \Phi X) & =K(X, \Phi X)+K(\Phi X, \xi)=K(X, \Phi X)+1 \\
\operatorname{Ric}_{g}(\xi, \xi) & =K(X, \xi)+K(\Phi X, \xi)=2
\end{aligned}
$$


So if $g$ is $\eta$-Einstein we have

$$
\operatorname{Ric}_{g}=\alpha g+(2-\alpha) \eta \otimes \eta,
$$

and this gives $K(X, \Phi X)=\alpha-1$.

Now if a Sasakian structure $\mathcal{S}=(\xi, \eta, \Phi, g)$ has constant $\Phi$-sectional curvature $c$, then it can be transformed to one of the cases $c=1,-3$, or -4 by a transverse homothety. The Uniformization Theorem can now be stated as:

Theorem 31 (Uniformization Bel00). Let $M$ be a 3-dimensional compact manifold admitting a Sasakian structure $\mathcal{S}=(\xi, \eta, \Phi, g)$. Then

(1) If $\mathcal{S}$ is positive, $M$ is spherical, and there is a Sasakian metric of constant $\Phi$-sectional curvature 1 in the same deformation class as $g$.

(2) If $\mathcal{S}$ is negative, $M$ is of $\widetilde{S L_{2}}$ type, and there is a Sasakian metric of constant $\Phi$-sectional curvature -4 in the same deformation class as $g$.

(3) If $\mathcal{S}$ is null, $M$ is nil, and there is a Sasakian metric of constant $\Phi$-sectional curvature -3 in the same deformation class as $g$.

In the positive case the universal cover $\tilde{M}$ is $S^{3}$ with its standard round sphere metric. In this case one generally needs both type I and II deformations. In the negative case $\tilde{M}=B^{2} \times \mathbb{R}$, the product of the unit 2-ball with $\mathbb{R}$; whereas, in the null case $\tilde{M}=\mathbb{R}^{3}$. In both of these cases the metrics have constant $\Phi$ sectional curvature, but not constant sectional curvature. Moreover, deformations of type I do not exist in the both negative and null cases except for $\mathcal{D}$-homotheties. Clearly, these 'standard metrics' are all $\eta$-Einstein. More generally in any dimension constant $\Phi$-sectional curvature metrics are $\eta$-Einstein.

In the positive case deformations of type I are definitely needed. Theorem 31 does not hold without them. A counterexample is the weighted Sasakian structure on $S^{3}$ with weights $\mathbf{w}=(p, q), p \neq 1$. The transverse space of the characteristic foliation is the weighted projective line $\mathbb{P}_{\mathbb{C}}^{1}(p, q)$ which is an example of a Fano orbifold which does not admit an orbifold metric of constant curvature. More generally, the existence of Kähler-Einstein metrics on weighted projective spaces of arbitrary dimension is obstructed by the non-vanishing of the Futaki character ACGTF04.

Remark 32. A Sasakian-Einstein structure on a 3-Sasakian manifold does not have to be a part of the 3-Sasakian structure. The simplest example when this is the case is the lens space $\mathbb{Z}_{k} \backslash S^{3}$. Consider the unit 3-sphere $S^{3} \simeq S p(1)$ as the unit quaternion $\sigma \in \mathbb{H}$. Such a sphere has two 3-Sasakian structures generated by the left and the right multiplication. Consider the homogeneous space $\mathbb{Z}_{k} \backslash S^{3}$, where the $\mathbb{Z}_{k}$-action is given by the multiplication from the left by $\rho \in S p(1), \rho^{k}=1$. The quotient still has the "right" 3-Sasakian structure. But it also has a "left" Sasakian structure (the centralizer of $\mathbb{Z}_{k}$ in $S p(1)$ is an $S^{1}$ and it acts on the coset from the left). This left Sasakian structure is actually regular while none of the Sasakian structures of the right 3-Sasakian structure can be regular unless $k=1,2$.

Example 33. Consider the standard metric on $S^{3} \longrightarrow S^{2}$ of constant sectional curvature equal to 1 . This metric is Sasakian-Einstein and 3-Sasakian. Applying $\mathcal{D}$-homothety to this metric produces a family of $\eta$-Einstein $\mathrm{U}(2)$-invariant metrics which can be written as $\sigma_{1}^{2}+\sigma_{2}^{2}+a \sigma_{3}^{2}$, where $\sigma_{1}, \sigma_{2}$ and $\sigma_{3}$ are the standard leftinvariant 1-forms on $S^{3} \simeq S U(2)$ and $a$ is a nonzero constant. The metrics were 
first considered by Berger. We will return to this example in the last section as it it the most basic Einstein-Weyl manifold.

Let us now consider some examples of Sasakian structures on 3-manifolds represented as links of isolated hypersurface singularities defined by weighted homogeneous polynomials.

Example 34. Positive Sasakian 3-manifolds. Belgun's Theorem 31 says that every positive Sasakian 3-manifold is a spherical space form $S^{3} / \Gamma$ where $\Gamma$ is a finite subgroup of $S U(2)$. These are precisely

(1) $\Gamma=\mathbb{Z}_{p}$ the cyclic group of order $p$,

(2) $\Gamma=\mathbb{D}_{m}^{*}$ a binary dihedral group where $m$ is an integer greater than 2 ,

(3) $\Gamma=\mathbb{T}^{*}$ the binary tetrahedral group,

(4) $\Gamma=\mathbb{O}^{*}$ the binary octahedral group,

(5) $\Gamma=\mathbb{I}^{*}$ the binary icosahedral group.

Orlik Orl70] shows that all of these groups can be realized by links of weighted homogeneous polynomials, and that this exhausts all cases with $|\mathbf{w}|>d$. It should be noted, however, that not all lens spaces $L(p, q)$ occur, but only $L(p, 1)$. The table below indicates the subgroup $\Gamma \subset S U(2)$ together with a representative polynomial and the corresponding Dynkin diagram of the singularity.

\begin{tabular}{|l|l|l|}
\hline Diagram & Subgroup & Polynomial \\
\hline \hline$A_{p-1}$ & $\mathbb{Z}_{p}$ & $z_{0}^{p}+z_{1}^{2}+z_{2}^{2}$ \\
\hline$D_{m}$ & $\mathbb{D}_{m}^{*}$ & $z_{0}^{2} z_{1}+z_{1}^{m}+z_{2}^{2}$ \\
\hline$E_{6}$ & $\mathbb{T}^{*}$ & $z_{0}^{4}+z_{1}^{3}+z_{2}^{2}$ \\
\hline$E_{7}$ & $\mathbb{O}^{*}$ & $z_{0}^{3}+z_{1}^{3} z_{0}+z_{2}^{2}$ \\
\hline$E_{8}$ & $\mathbb{I}^{*}$ & $z_{0}^{5}+z_{1}^{3}+z_{2}^{2}$ \\
\hline
\end{tabular}

Notice that $L(5,3,2)=S^{3} / \mathbb{I}^{*}$ is the famous Poincaré homology sphere.

Example 35. Null Sasakian 3-manifolds. Up to covering these are all non-trivial circle bundles over a complex torus, and it is known Mil75 that all such bundles can be constructed from the three dimensional Heisenberg group $H \simeq \mathbb{R}^{3}$ which can be represented as a subgroup of $G L(3, \mathbb{R})$ by considering matrices of the form

$$
\mathbb{A}=\left(\begin{array}{ccc}
1 & x & z \\
0 & 1 & y \\
0 & 0 & 1
\end{array}\right) ; x, y, z \in \mathbb{R} .
$$

We also define subgroups $H_{k} \subset H$ of matrices for which $x, y, z$ are integers divisible by some positive integer $k$. It is easy to see that the quotient manifold $M_{k}=H / H_{k}$ is a circle bundle over a torus with Chern number $\pm k$. The fibration $\pi: M_{k} \longrightarrow T^{2}$ is explicitly given by

$$
\pi(\mathbb{A})=(x \bmod k, z \bmod k) .
$$

Now Sasaki Sas65 showed that $\mathbb{R}^{3}$ has a natural null Sasakian $\eta$-Einstein metric with constants $(\lambda, \nu)=(-2,4)$, although the connection with the Heisenberg group wasn't observed until later. This metric is

$$
g=d x^{2}+d y^{2}+(d z-y d x)^{2} .
$$


The Sasakian structure $\mathcal{S}=(\xi, \eta, \Phi, g)$ on $H$ is defined by the formulas

$$
\left\{\begin{array}{l}
\eta=d z-y d x \\
\Phi=\left(\frac{\partial}{\partial x}+y \frac{\partial}{\partial z}\right) \otimes d y-\frac{\partial}{\partial y} \otimes d x .
\end{array} \quad \xi=\frac{\partial}{\partial z}\right.
$$

Now one can easily check that the tensor fields defining $\mathcal{S}$ are invariant under the action of $H$ on itself. Hence, $\mathcal{S}$ defines a Sasakian structure on the compact quotient manifolds $M_{k}$. Milnor Mil75 observed that the integral homology can easily be computed, viz.

$$
H_{1}\left(M_{k}, \mathbb{Z}\right)=H_{k} /\left[H_{k}, H_{k}\right]=\mathbb{Z} \oplus \mathbb{Z} \oplus \mathbb{Z}_{k} .
$$

However, as indicated by the table below only the cases $k=1,2,3$ can be realized as links of isolated hypersurface singularities of weighted homogeneous polynomials. There are precisely three null Sasakian 3-manifolds that can be represented by links of isolated hypersurface singularities of weighted homogeneous polynomials Orl70. These are realized by arbitrary weighted homogeneous polynomials of degrees 6,4 and 3 , and they exhaust all possibilities that satisfy $|\mathbf{w}|=d$. The table below gives the weights, degrees, and number of monomials of the three polynomials.

\begin{tabular}{|c|c|c|c|}
\hline manifold & weight vector & degree & \# of monomials \\
\hline \hline$M_{1}$ & $(1,2,3)$ & 6 & 7 \\
\hline$M_{2}$ & $(1,1,2)$ & 4 & 9 \\
\hline$M_{3}$ & $(1,1,1)$ & 3 & 10 \\
\hline
\end{tabular}

Example 36. Negative Sasakian 3-manifolds. These are up to covering circle bundles over Riemann surfaces of genus $g>1$. There are infinitely many examples which can be represented as links of isolated hypersurface singularities defined by weighted homogeneous polynomials. Milnor proves Mil75 that in the case of Brieskorn 3-manifolds $L\left(a_{0}, a_{1}, a_{2}\right)=\widetilde{S L}(2, \mathbb{R}) / \Gamma$, where $\widetilde{S L}(2, \mathbb{R})$ is the universal cover of $S L(2, \mathbb{R})$ and $\Gamma$ is a discrete subgroup completely determined by the triple $\left(a_{1}, a_{2}, a_{3}\right)$. Perhaps the most interesting are the integral homology spheres. In this case a theorem of Saeki Sae87 says that the link of an isolated hypersurface singularity defined by a weighted homogeneous polynomial has the same knot type as a Brieskorn link. Now Breiskorn's graph Theorem Bri66 implies that a Brieskorn link $L(\mathbf{a})$ is a homology sphere if and only if $\operatorname{gcd}\left(a_{i}, a_{j}\right)=1$ for all $i \neq j=0,1,2$. So clearly 3-dimensional homology spheres are quite numerous, and all but one, the Poincaré homology sphere $L(5,3,2)$, have infinite fundamental group. More, generally for any link of an isolated hypersurface singularity defined by a weighted homogeneous polynomial, Orlik Orl70 proved that $\pi_{1}$ is infinite if and only if $d \geq|\mathbf{w}|$, and $\pi_{1}$ is infinite nilpotent if and only if $d=|\mathbf{w}|$ which is the Euclidean or null Sasakian case. Obviously, for integral homology spheres $\pi_{1}$ must be perfect, i.e. $\left[\pi_{1}, \pi_{1}\right]=\pi_{1}$ where $\left[\pi_{1}, \pi_{1}\right]$ denotes the commutator subgroup. A rather large class of examples can be treated by considering Brieskorn polynomials of the form

$$
f=z_{0}^{6 k \pm 1}+z_{1}^{3}+z_{2}^{2} .
$$

Since $d-|\mathbf{w}|=6 k-6 \pm 1 \geq 0, \pi_{1}(L(6 k \pm 1,3,2))$ is infinite in all cases except the Poincaré homology sphere $S^{3} / I^{*}=L(5,3,2)$. For example, $L(7,3,2)$ is the quotient of the universal cover $\widetilde{S L}(2, \mathbb{R})$ of $S L(2, \mathbb{R})$ by a co-compact discrete subgroup $\Gamma \subset \widetilde{S L}(2, \mathbb{R})$, and there is a covering of $L(7,3,2)$ by the total space of a nontrivial circle bundle over a Riemann surface of genus $g=3$ Mil75. More generally, except 
for the Poincaré homology sphere, $L(6 k \pm 1,3,2)$ has a finite covering by a manifold that is diffeomorphic to a nontrivial circle bundle over a Riemann surface of some genus $g>1$.

We end this section with a brief discussion of how one can distinguish integral homology spheres in three dimensions. This can be done by the Casson invariant $\lambda$ which roughly speaking counts the number of irreducible representations of $\pi_{1}$ in $S U(2)$. More explicitly for a Brieskorn homology sphere $L(p, q, r)$ Fintushel and Stern [FS90] proved that $\lambda(L(p, q, r))$ equals $-\frac{1}{2}$ times the number of conjugacy classes of irreducible representations of $\pi_{1}(L(p, q, r))$ into $S U(2)$. Furthermore, they showed how $\lambda(L(p, q, r))$ can be computed from the Hirzebruch signature of the parallelizable manifold $V(p, q, r)$ whose boundary is the link $L(p, q, r)$. Explicitly,

$$
\lambda(L(p, q, r))=\frac{\operatorname{sig} V(p, q, r)}{8} .
$$

Now Brieskorn Bri66 had computed the signature of $V(6 k-1,3,2)$ to be $-8 k$, giving $\lambda(L(6 k-1,3,2))=-k$. Thus, $L(6 k-1,3,2)$ give an infinite sequence of inequivalent homology spheres. Moreover, it is known that $\lambda(L(7,3,2))=-1$, and since $k=1$ from the previous sequence is the Poincaré homology sphere, which is clearly distinct, we see that $L(7,3,2)$ is also distinct from the others. For further discussion and references we refer the reader to the recent book Sav02].

\section{8. $\eta$-Einstein Metrics in Dimension 5 And Higher}

In this section we give a large number of examples of manifolds in dimension five and higher that admit Sasakian $\eta$-Einstein metrics. For null and negative Sasakian structures the proof of existence amounts to presenting examples as links of weighted homogeneous polynomials and then invoking Theorem 28 In the positive or Fano case the existence of Sasakian $\eta$-Einstein metrics is equivalent to the existence of Sasakian-Einstein metrics which has been studied quite extensively BGN03a, BGK03, BGKT03, Kol04b.

The list of spin 5-manifolds which admit many inequivalent families of SasakianEinstein metrics is now long and keeps growing. For example, $S^{5}$ has at least 68 inequivalent Sasakian-Einstein metrics realized as Brieskorn-Pham links. The example below gives a subfamily.

Example 37. Consider sequences of the form $\mathbf{a}=(2,3,7, m)$. By explicit calculation, the corresponding link $L(\mathbf{a})$ gives a Sasakian-Einstein metric on $S^{5}$ if $5 \leq m \leq 41$ and $m$ is relatively prime to at least two of $2,3,7$. This is satisfied in 27 cases.

Recently, Kollár has shown that there are many inequivalent Sasakian-Einstein metrics on all $k$-fold connected sums of $S^{2} \times S^{3}$ Kol04b. On the other hand Boyer and Galicki showed that BG03

Theorem 38. The links $L(3,3,3, k) k>2, L(2,4,4, p), p>2$ and $L(2,3,6, m)$, $m>4$ all admit Sasakian-Einstein structures.

The above links are rational homology 5 -spheres under suitable assumptions on $k, p, m$. For example $L(3,3,3, k)$ is a rational homology sphere with $H_{2}(L, \mathbb{Z})=$ $\mathbb{Z}_{k} \oplus \mathbb{Z}_{k}$ as long as $k$ is prime to 3 . Since any $L(3,3,3, k)$ is spin, by Smale's theorem Sma62, the second homology group completely determines diffeomorphism type of $L(3,3,3, k)$. 
In the null case there appear to be only a finite number of manifolds admitting such structures. We begin with a non-existence result in dimension 5 .

Theorem 39. Let $\mathcal{S}$ be a null Sasakian structure on a compact 5-manifold with $H_{1}(M, \mathbb{Z})=0$. Then $2 \leq b_{2}(M) \leq 21$ and $H_{2}(M, \mathbb{Z})$ is torsion free. Furthermore, if $b_{2}(M)=21$ then $\mathcal{S}$ is regular and $M$ is diffeomorphic to $\# 21\left(S^{2} \times S^{3}\right)$, and $M / \mathcal{F}_{\xi}$ is a K3 surface.

Proof. The lower bound on $b_{2}(M)$ is immediate from Corollary 1.10 of [BGN03a], while the upper bound as well as the last statement follow from Corollary 81 of Kol04a. That $H_{2}(M, \mathbb{Z})$ is torsion free follows from Proposition 80 of Kol04a.

We mention that the lower bound in Theorem 39 holds with the weaker condition $H_{1}(M, \mathbb{R})=0$. We now have

Corollary 40. $S^{5}$ and $S^{2} \times S^{3}$ or any quotient by a finite group do not admit null Sasakian structures.

Compact 5-manifolds with null Sasakian structures can be easily obtained from the list of 95 orbifold K3 surfaces.

Example 41. In 1979 Reid Rei80 produced a list of 95 weighted K3 surfaces given as well-formed hypersurfaces in weighted projective space $\mathbb{P}\left(w_{0}, w_{1}, w_{2}, w_{3}\right)$. His result generalizes the standard construction of the K3 surface as a quartic in $\mathbb{C P}(3)$. With each example of Reid one can consider the associated 5-dimensional link $L_{f}$. By a result of Boyer and Galicki BG01b all $L_{f}$ are diffeomorphic to some $k$-fold connected sum of $S^{2} \times S^{3}$, where $k=b_{2}\left(L_{f}\right)$ can be computed in each case by the Milnor-Orlik procedure [MO70]. For instance $L(4,4,4,4) \simeq \# 21\left(S^{2} \times S^{3}\right)$ is regular and it is a circle bundle over the $K 3$-surface $X_{4} \subset \mathbb{P}^{3}$. No.3 On Reid's list in the table of [IF00] is also a BP link $L(6,6,6,2) \simeq \# 21\left(S^{2} \times S^{3}\right)$. The degree 6 surface in $X_{6} \subset \mathbb{P}(1,1,1,3)$ given by vanishing of $f(\mathbf{z})=z_{0}^{6}+z_{1}^{6}+z_{2}^{6}+z_{3}^{2}$ is easily seen to be non-singular and as a result we also get a regular null Sasakian structure on a K3-surface and regular null Sasakian structure on the corresponding circle bundle. However, $X_{4}$ and $X_{6}$ cannot be the same as complex algebraic varieties and $L(4,4,4,4)$ and $L(6,6,6,2)$ are not equivalent as Sasakian manifold. In all, there are 11 more examples with are Brieskorn-Pham links. All Reid's examples have $3 \leq b_{2}\left(L_{f}\right) \leq 21$. Hence, for instance, the link $L_{f}$ with

$$
f(\mathbf{z})=z_{0}^{2}+z_{1}^{2}+z_{2} z_{0} z_{1} z_{3}+z_{2}^{2} z_{3}+z_{2}^{3} z_{0}+z_{3}^{3}
$$

must be diffeomorphic to $\# 3\left(S^{2} \times S^{3}\right)$. No. 14 on the list, is a BP link $L(2,3,12,12)$ and it diffeomorphic to $\# 20\left(S^{2} \times S^{3}\right)$. It is interesting that $b_{2}(L)=17$ does not occur on the Reid's list. But other examples can be considered. For instance, Fletcher gives a list of 84 examples of codimension 2 complete intersections IF00. On the other hand, the only codimension 3 weighted complete intersection is the intersection of 3 quadrics in $\mathbb{P}^{5}$.

By writing a simple Maple program we have computed the second Betti number of all 95 orbifold K3 surfaces, and we find

Corollary 42. \#k(S $\left.S^{2} \times S^{3}\right)$ admit both positive and null Sasakian $\eta$-Einstein structures for $3 \leq k \leq 21$ and $k \neq 17$. 
The question which of the simply connected compact spin 5-manifolds of Smale's list admit null Sasakian structures is equivalent to classifying all orbifold K3surfaces. This is still open. An interesting question is whether $k=2$ or 17 can occur. In higher dimensions there are many, though probably finite, null Sasakian structures, and they will all admit $\eta$-Einstein null structures. Interesting examples include the over 6000 Calabi-Yau orbifolds in complex dimension 3 [CLS90].

In the negative case even just Brieskorn-Pham links produce already very many examples. The simplest ones are the Fermat hypersurfaces of degree $d$ in $\mathbb{P}^{3}$. For $d=4$ it gives \#21 $\left(S^{2} \times S^{3}\right)$ as discussed in Example 41 above. For $d \geq 5$ we get negative Sasakian $\eta$-Einstein metrics on $\# k\left(S^{2} \times S^{3}\right)$ for $k=(d-2)\left(d^{2}-2 d+2\right)+1$. These begin with $k=52$ and grow rapidly. To obtain negative Sasakian $\eta$-Einstein structures we turn to some different examples. First, such structures exist on rational homology spheres, and in particular on $S^{5}$.

Example 43. Consider $L(k, k, k+1, p)$. Such a link is negative as long as $k, p>3$ or with $k=3$ and $p>12$. Moreover, $L(k, k, k+1, p)$ is a 5-sphere as long as $p$ is prime to both $k$ and $k+1$ which can easily be arranged.

Proposition 44. Every negative Sasakian structure on $S^{5}$ is non-regular and there exists infinitely many inequivalent negative Sasakian $\eta$-Einstein structures. Hence, $S^{5}$ admits infinitely many different Lorentzian Sasakian-Einstein structures.

Proof. The existence of infinitely many non-regular negative Sasakian structures on $S^{5}$ follows immediately from Example 43. These lie in different deformation classes, and from Theorem [17 each deformation class has a negative $\eta$-Einstein metric. To prove the first statement we assume that $S^{5}$ has a regular negative Sasakian structure. Then we have a circle bundle $S^{5} \longrightarrow X$ with $c_{1}(X)$ negative, $\pi_{1}(X)=\{1\}$, and $b_{2}(X)=1$. This is impossible by a Theorem of Yau, cf. Theorem V.1.1 of [BPVdV84].

It is rather easy to produce examples of negative Sasakian $\eta$-Einstein structures on $\# k\left(S^{2} \times S^{3}\right)$; however, we haven't been able to find one nice sequence which does this, or even a sequence that works for odd $k$. The examples below give the essential ideas.

Example 45. This is example 43 with $p=k+1$. Now the BP link is $L(k, k, k+1, k+$ 1 ) and it is easy to see that the induced Sasakian structures are negative as long as $k \geq 4$. Here the Milnor-Orlik method gives $b_{2}(L(k, k, k+1, k+1))=k(k-1)$. This gives negative Sasakian $\eta$-Einstein structures on $\# k\left(S^{2} \times S^{3}\right)$ for infinitely many $k$, but with larger and larger gaps as $k$ grows. For low values of $k$, for example, $k=4$ and 5, we get negative Sasakian structures on $\# 12\left(S^{2} \times S^{3}\right)$, and $\# 20\left(S^{2} \times S^{3}\right)$, respectively.

Example 46. Consider the BP links $L(p, q, r, p q r)$ such that $p, q, r \in \mathbb{Z}^{+}$are pairwise relatively prime. The transverse space is a surface $X_{p q r} \subset \mathbb{P}(p r, q r, p q, 1)$ and it is automatically well-formed, i.e., it has only isolated orbifold singularities. Hence, $L(p, q, r, p q r)$ is diffeomorphic to $\# k\left(S^{2} \times S^{3}\right)$, where

$$
k=b_{2}(L(p, q, r, p q r))=(p q r-p q-p r-q r-1)+p+q+r .
$$

Now, $L(p, q, r, p q r)$ is negative when the term in parenthesis is positive, i.e. when $p q+p r+q r+1<p q r$. To simplify we can consider $L(2,3, r, 6 r)$ with $r$ prime to both 3 and 2. $L(2,3,7,42)$ is one of the examples on Reid's list. But from $r>7$ we 
get infinitely many examples with second Betti number $b_{2}=2(r-1)$. For example for $r=11$, we see that $L(2,3,11,66)$ is diffeomorphic to $\# 20\left(S^{2} \times S^{3}\right)$.

Notice that both Examples 45 and 46 produce $\eta$-Einstein metrics on $\# k\left(S^{2} \times S^{3}\right)$ for even $k$ only. We haven't yet found a nice series that gives metrics for odd values of $k$. However, we can obtain $\eta$-Einstein metrics for odd $k$ if we consider the more general weighted homogeneous polynomials.

Example 47. Here we give a few examples only to illustrate the method. A much more extensive list can be generated with the aid of a computer a la JK01, BGN03b. We begin with two inequivalent 'twin' Sasakian $\eta$-Einstein metrics on $S^{2} \times S^{3}$. Consider the weighted homogeneous polynomials

$$
z_{0}^{21} z_{1}+z_{1}^{5} z_{2}+z_{2}^{3} z_{0}+z_{3}^{2}, \quad z_{0}^{21} z_{1}+z_{1}^{5} z_{0}+z_{2}^{3} z_{1}+z_{3}^{2} .
$$

Both have degree 316 and both give links diffeomorphic to $S^{2} \times S^{3}$. The weight vectors are $\mathbf{w}=(13,43,101,158)$ and $\mathbf{w}=(11,61,85,158)$, respectively. So $|\mathbf{w}|-$ $d=-1$ giving negative Sasakian $\eta$-Einstein metrics on $S^{2} \times S^{3}$.

Similarly, the polynomials

$$
z_{0}^{20}+z_{1}^{3} z_{3}+z_{2}^{3} z_{1}+z_{3}^{2} z_{0} \quad z_{0}^{10}+z_{1}^{4} z_{2}+z_{2}^{2} z_{3}+z_{3}^{2} z_{0}
$$

have degree 40 and 159, respectively. They give negative Sasakian $\eta$-Einstein metrics on $\# 7\left(S^{2} \times S^{3}\right)$ and $\# 2\left(S^{2} \times S^{3}\right)$.

We have

Corollary 48. \#7( $\left.S^{2} \times S^{3}\right)$, \#12( $\left.S^{2} \times S^{3}\right)$ and $\# 20\left(S^{2} \times S^{3}\right)$ all admit positive, null, and negative Sasakian $\eta$-Einstein structures.

It is easy to construct examples of negative Sasakian $\eta$-Einstein metrics on homotopy spheres in arbitrary odd dimensions.

Example 49. Let the integers $r_{i}, i=1, \ldots, 2 m$ be any pairwise prime positive integers. Then the $(4 m+1)$-dimensional link $L\left(2,2 r_{1}, \ldots, 2 r_{2 m}, a\right)$ is diffeomorphic to the standard sphere if $a \equiv \pm 1 \bmod 8$ and to the Kervaire sphere if $a \equiv \pm 3$ mod 8. The link will be negative when $\sum_{i} \frac{1}{r_{i}}<\frac{a-2}{a}$ which is easily satisfied for $r_{i}$ 's large enough.

Corollary 50. In any dimension $4 m+1$ both the standard and the Kervaire spheres admit infinitely many inequivalent negative Sasakian $\eta$-Einstein structures.

In dimension $4 m+3$ one can construct examples of negative Sasakian structures should exists on all homotopy spheres which bound parallelizable manifolds. That is to say, all BP links that by Brieskorn Graph Theorem are homeomorphic to a sphere of dimension $4 m+3$ and are negative should easily contain all possible oriented diffeomorphism types. We have checked it in dimension 7 with the following

Theorem 51. All 28 oriented diffeomorphism types of homotopy 7-spheres admit negative Sasakian structures. Hence, they all admit Lorentzian Sasakian-Einstein structures.

Proof. Consider $L(k, k, k, k+1, p)$, where $p$ is prime to both $k$ and $k+1$. The link $L$ is a homotopy 7 -sphere and it is negative for $k$ and $p$ large enough. Using the computer codes of BGKT03, it is easy to check that such links realize all 28 oriented diffeomorphism types. 
Similarly, one should be able to show that the homotopy spheres in dimensions 11 and 15 that bound parallelizable manifolds admit negative Sasakian structures.

Remark 52. Many of the Sasakian $\eta$-Einstein structures admit large moduli spaces. This is particularly true for BP type polynomials. We refer the reader to our previous work BGN03b, BGK03, BGKT03].

\section{Positive $\eta$-Einstein Structures and Einstein-Weyl Geometry}

In this chapter we investigate relations between Einstein-Weyl structures and $\eta$-Einstein metrics. The first insight into this relation was provided by Swann and Pedersen PS93 and by Higa Hig93 who studied Einstein-Weyl geometries of circle bundles over positive Kähler-Einstein manifolds. Later Narita in a series of papers made the connection to $\eta$-Einstein geometry more explicit Nar93, Nar97, Nar98. We begin with some basic facts about Einstein-Weyl geometry (see CP99 for a review of the subject).

Definition 53. A Weyl structure on a manifold $M$ of dimension $n \geq 3$ is defined by a pair $\mathcal{W}=([g], D)$, where $[g]$ is a conformal class of Riemannian metrics and $D$ is the unique torsion-free connection preserving $[g]$. The connection $D$ is called the Weyl connection.

Then, for every Riemannian metric $g$ in the conformal class, there exists a 1-form $\theta$, uniquely determined by $D$ and $g$, such that, for every $X, Y \in \mathcal{X}(M)$,

$$
D_{X} Y=\nabla_{X} Y+\theta(X) Y+\theta(Y) X-g(X, Y) \zeta,
$$

where $\nabla$ denotes the Levi-Civita connection of $g$ and $\theta^{\#}$ the dual vector field of $\theta$ with respect to $g$. In particular we have

$$
D g=-2 \theta \otimes g .
$$

The above condition is invariant under Weyl transformation, i.e.,

$$
g^{\prime}=e^{2 f} g, \quad \theta^{\prime}=\theta+d f, \quad f \in C^{\infty}(M)
$$

Hence, choosing a Riemannian metric in $[g]$ we sometimes abuse the terminology and refer to the pair $\mathcal{W}=(g, \theta)$ as a Weyl structure. It is known that on a compact, conformal manifold of dimension at least 3 , the conformal class $[g]$ contains a unique (up to homothety) metric $g$ such that the corresponding form $\theta$ is $g$-co-closed (cf. Gau95). This metric is the Gauduchon metric or the Gauduchon gauge.

Definition 54. A conformal manifold $(M,[g], D)$ is called Einstein-Weyl if

$$
\operatorname{Ric}^{D}(X, Y)+\operatorname{Ric}^{D}(Y, X)=\Lambda g(X, Y)
$$

for some smooth function $\Lambda$ on $M$.

Clearly the Einstein-Weyl condition on the symmetrized Ricci tensor of $D$ is conformally invariant. A Weyl structure is said to be closed (resp. exact) if the Weyl connection is locally (resp. globally) the Levi-Civita connection of a compatible metric. Hence a closed Einstein-Weyl structure admits local (but not necessarily global) compatible Einstein metrics.

On compact manifolds of dimension at least 3 with a closed but not exact Einstein-Weyl structure the form $\theta$ of the Gauduchon metric $g$ is $g$ parallel: $\nabla \theta=0$ Gau95. In particular, $\theta$ is closed, hence $g$-harmonic. 
On an almost contact metric manifold $(M, \xi, \eta, \Phi, g)$ it is natural to ask whether $M$ has an Einstein-Weyl structure $\mathcal{W}=(g, \theta)$ such that $\theta=f \eta$, for some function $f \in C^{\infty}(M)$, and whether the metric $g$ compatible with the almost contact structure is the Gauduchon metric of $[g]$. In fact, if $(M, \xi, \eta, \Phi, g)$ is an almost contact manifold then, for any 1 -form $\theta$, the Weyl structure $\mathcal{W}=(g, \theta)$ on $M$ is connected to $\mathcal{S}=(\xi, \eta, \Phi, g)$ by several fundamental relations. The existence of Einstein-Weyl structures on almost contact metric manifolds have been considered by several authors [Mat02, [Mat00], Nar97], Nar98. Here we review and then extend some of the known results.

Let $(M, \xi, \eta, \Phi, g)$ be a $(2 n+1)$-dimensional, $n \geq 1$, almost contact metric manifold. If $\mathcal{W}=(g, \theta)$ is a Weyl structure on $M$, for every $X, Y \in \mathcal{X}(M)$, the Ricci tensor $\operatorname{Ric}^{D}$ of $D$ and $\operatorname{Ric}_{g}$ of $\nabla$ are related by the following equation Hig93:

$$
\begin{aligned}
& \operatorname{Ric}^{D}(X, Y)=\operatorname{Ric}_{g}(X, Y)-2 n\left(\nabla_{X} \theta\right)(Y)+\left(\nabla_{Y} \theta\right)(X)+ \\
& +(2 n-1) \theta(X) \theta(Y)+\left(\delta \theta-(2 n-1)|\theta|^{2}\right) g(X, Y),
\end{aligned}
$$

where $\delta \theta,|\theta|$ are the codifferential and the point-wise norm of $\theta$ with respect to $g$. The following local characterizations of Einstein-Weyl structures can be found in Hig93.

Proposition 55. Let $\mathcal{W}=(g, \theta)$ be a Weyl structure on an almost contact metric manifold $(M, \xi, \eta, \Phi, g)$ of dimension $2 n+1 \geq 3$. Then $\mathcal{W}=(g, \theta)$ is an EinsteinWeyl structure if and only if there exists a smooth function $\sigma$ on $M$ such that:

(33) $\frac{1-2 n}{2}\left(\left(\nabla_{X} \theta\right)(Y)+\left(\nabla_{Y} \theta\right)(X)\right)+(2 n-1) \theta(X) \theta(Y)+\operatorname{Ric}_{g}(X, Y)=\sigma g(X, Y)$, for every $X, Y \in \mathcal{X}(M)$.

Proposition 56. Let $(M, \xi, \eta, \Phi, g)$ be an almost contact metric manifold of dimension $2 n+1 \geq 3$ and $\theta$ a 1-form on $M$. Consider the Weyl structures $\mathcal{W}^{ \pm}$ defined by $\mathcal{W}^{ \pm}=(g, \pm \theta)$. Then both $\mathcal{W}^{+}$and $\mathcal{W}^{-}$are Einstein-Weyl if and only if $(g, \theta)$ satisfies the following two equations for every $X, Y \in \mathcal{X}(M)$ :

$$
\begin{gathered}
\left(\nabla_{X} \theta\right)(Y)+\left(\nabla_{Y} \theta\right)(X)+\frac{2}{2 n+1} \delta \theta g(X, Y)=0 \\
\operatorname{Ric}_{g}(X, Y)-\frac{s}{2 n+1} g(X, Y)=\frac{2 n-1}{2 n+1}|\theta|^{2} g(X, Y)-(2 n-1) \theta(X) \theta(Y),
\end{gathered}
$$

where $s$ is the scalar curvature of $g$.

First of all, as a direct consequence of (33), we can state

Proposition 57. Let $(M, \xi, \eta, \Phi, g)$ be a $K$-contact metric manifold of dimension $2 n+1 \geq 3$ and suppose that $M$ admits an Einstein-Weyl structure $W=(g, \theta)$ with $\theta=f \eta$ for some non constant function $f$ on $M$. Then $X(f)=0$ for every vector field $X$ on $M$ such that $\eta(X)=0$ and the Ricci tensor of $M$ is given by $\operatorname{Ric}_{g}=\sigma g-(2 n-1)\left(f^{2}-\xi(f)\right) \eta \otimes \eta$.

Proof. We recall, at first, that the Ricci tensor of a $K$-contact manifold always satisfies the relation $\operatorname{Ric}_{g}(X, \xi)=2 n \eta(X)$. Then, for every $X$ horizontal vector 
field on $M$ (here and in the following we shall call horizontal any vector field on $M$ orthogonal to $\xi$ ) and $Y=\xi$, (33) becomes

$$
\left(\nabla_{X} f \eta\right)(\xi)+\left(\nabla_{\xi} f \eta\right)(X)=0
$$

which easily implies the first part of the proposition. A straightforward computation proves the second part of the assert.

Corollary 58. If a $K$-contact manifold $M$ of dimension at least 5 admits an Einstein-Weyl structure $\mathcal{W}=(g, f \eta)$ with $f \in C^{\infty}(M)$, then $M$ is $\eta$-Einstein. In particular, in such case, $\sigma$ and $f^{2}-\xi(f)$ must be constant.

The following example generalizes the 3-dimensional case of Example 35.

Example 59. Let $H(n)$ be the Heisenberg Lie group

$$
H(n)=\left\{\left(\begin{array}{ccc}
1 & \mathbf{x}^{t} & z \\
0 & 1 & \mathbf{y} \\
0 & 0 & 1
\end{array}\right) ; \mathbf{x}, \mathbf{y} \in \mathbb{R}^{n}, z \in \mathbb{R}\right\},
$$

and let $g$ be the following left invariant metric on $H(n)$

$$
g=d \mathbf{x} \cdot d \mathbf{x}+d \mathbf{y} \cdot d \mathbf{y}+(d z-\mathbf{x} \cdot d \mathbf{y})^{2} .
$$

The Sasakian structure $\mathcal{S}=(\xi, \eta, \Phi, g)$ on $H(n)$ is defined by the formulas

$$
\left\{\begin{array}{l}
\eta=d z-\mathbf{x} \cdot d \mathbf{y}, \quad \xi=\frac{\partial}{\partial z} \\
\Phi=\sum_{i}\left(\left(\frac{\partial}{\partial x^{i}}+y^{i} \frac{\partial}{\partial z}\right) \otimes d y^{i}-\frac{\partial}{\partial y^{i}} \otimes d x^{i}\right) .
\end{array}\right.
$$

One can easily check that this is a null Sasakian $\eta$-Einstein structure on $H(n)$ Sas65. Moreover, a transverse homothety of $\mathcal{S}$ by a constant $a$ gives a null Sasakian $\eta$-Einstein structure $\overline{\mathcal{S}}=(\bar{\xi}, \bar{\eta}, \bar{\Phi}, \bar{g})$ which admits the Einstein-Weyl structure $\mathcal{W}=$ $(\bar{g}, f \bar{\eta})$, with $f=\alpha \tan (z+c), \alpha, c \in \mathbb{R}$, provided that $a=\frac{1}{\alpha}$, and $\alpha>0$ such that $\alpha^{2}=\frac{2 n+2}{2 n-1}$. This generalizes to arbitrary odd dimension the Einstein-Weyl structure described in Nar97, Nar98. In addition, one can get compact examples by taking quotients of $H(n)$ by discrete subgroups.

From the formula (33) we can also deduce that, if $\xi$ is a Killing vector field on the almost contact metric manifold $(M, \xi, \eta, \Phi, g)$ and $M$ admits an Einstein-Weyl structure $\mathcal{W}^{+}=(g, \mu \eta)$ for some constant $\mu$, then $M$ admits the Einstein-Weyl structure $\mathcal{W}^{-}=(g,-\mu \eta)$ too. More precisely, applying the Proposition [56] to the $K$-contact manifolds, we obtain the following

Theorem 60. Let $(M, \xi, \eta, \Phi, g)$ be a K-contact manifold of dimension $2 n+1 \geq 3$ and $\theta$ a 1-form on $M$. Suppose that both $\mathcal{W}^{ \pm}=(g, \pm \theta)$ are Einstein-Weyl structures on $M$. Then $\theta$ is co-closed, but not closed, so $g$ is the Gauduchon metric, the dual vector field $\theta^{\#}$ to $\theta$ is a Killing vector field, and either

(1) $\theta(\xi)=0$ or

(2) $\theta=\mu \eta$ with $\mu \in \mathbb{R}$.

Proof. In fact, if both $\mathcal{W}^{ \pm}=(g, \pm \theta)$ are Einstein-Weyl structures on $(M, \xi, \eta, \Phi, g)$, the 1-form $\theta$ satisfies the both equations of Proposition [56]. In particular, substituting $\xi$ for $Y$ in (35) we get

$$
2 n \eta(X)-\frac{s}{2 n+1} \eta(X)=\frac{2 n-1}{2 n+1}|\theta|^{2} \eta(X)-(2 n-1) \theta(X) \theta(\xi) .
$$


Then the relation

$$
\theta(X) \theta(\xi)=0
$$

is true for every horizontal vector field $X$. Now define the set

$$
S=\left\{x \in M \mid \theta(X)_{x}=0 \forall \text { horizontal } X\right\} .
$$

$S$ is closed, and by (41) $\theta(\xi)=0$ on the open set $M \backslash S$. Then putting $X=Y=\xi$ in (34) implies that $\delta \theta=0$ on $M \backslash S$. But then again (34) implies $\left(\nabla_{X} \theta\right)(Y)+$ $\left(\nabla_{Y} \theta\right)(X)=0$ on $M \backslash S$ for any $X, Y \in \mathcal{X}(M)$. This means that the dual vector field $\theta^{\#}$ is a Killing field on $M \backslash S$. Consequently, if $\theta$ were closed on $M \backslash S$, it would also be parallel with respect to the Levi-Civita connection $\nabla$ of $g$. In particular for every $X \in \mathcal{X}(M)$ we have

$$
0=\left(\nabla_{X} \theta\right)(\xi)=-\theta(\Phi(X))
$$

implying that $\theta$ identically vanishes on $M \backslash S$. This proves that $\theta$ cannot be closed on $M \backslash S$.

On the other hand, the set $S_{0}$ where $\theta(\xi) \neq 0$ is open and a subset of $S$ by (41). Thus, $\theta=\mu \eta$ on $S$ for some $\mu \in C^{\infty}(S)$. So, for $X, Y$ horizontal vector fields (34) becomes

$$
\mu\left(\left(\nabla_{X} \eta\right)(Y)+\left(\nabla_{Y} \eta\right)(X)\right)+\frac{2}{2 n+1} \delta \theta g(X, Y)=0
$$

on $S$. But the first term vanishes since $\xi$ is a Killing vector field. This implies that $\delta \theta=0$ on $S$ and hence, on all of $M$. But then (34) gives $\left(\nabla_{X} \theta\right)(Y)+\left(\nabla_{Y} \theta\right)(X)=0$, on all $M$ for all $X, Y \in \mathcal{X}(M)$ which means that $\theta^{\#}$ is a Killing vector field, and also implies that $\mu$ is a constant. This completes the proof of the theorem.

We now discuss several corollaries of Theorem 60 First combining our results with Gau95 we have

Corollary 61. Let $(M, \xi, \eta, \Phi, g)$ be a compact $K$-contact manifold of dimension $2 n+1 \geq 3$ and $\theta$ a 1-form on $M$. Given the Weyl structure $\mathcal{W}=(g, \theta)$, then the metric $g$ is the Gauduchon metric of the conformal class $[g]$ if and only if $M$ admits both $\mathcal{W}^{ \pm}=(g, \pm \theta)$ as Einstein-Weyl structures.

Corollary 62. Let $(M, \xi, \eta, \Phi, g)$ be a K-contact manifold of dimension $2 n+1 \geq 5$. Then $(M, \xi, \eta, \Phi, g)$ admits an Einstein-Weyl structure $\mathcal{W}=(g, \theta)$ with $\theta=\mu \eta$, $\mu \in \mathbb{R}$, if and only if $M$ is $\eta$-Einstein with Einstein constants $\lambda, \nu$ such that $\nu<0$.

Proof. If $(M, \xi, \eta, \Phi, g)$ admits an Einstein-Weyl structure with 1-form $\theta=\mu \eta$ for some constant $\mu$, then from (33) we obtain

$$
\operatorname{Ric}_{g}=\sigma g-(2 n-1) \mu^{2} \eta \otimes \eta,
$$

which proves the only if part of the assertion. On the contrary, if we suppose that the Ricci tensor of $M$ is given by

$$
\operatorname{Ric}_{g}=\lambda g+\nu \eta \otimes \eta
$$

where $\nu<0$, (33) is satisfied for $\sigma=\lambda$ and $\theta=\mu \eta$ where $\mu$ is a constant such that $\mu^{2}=-\frac{\nu}{2 n-1}$.

In the case of Sasakian structures Theorem 60 can be improved: 
Theorem 63. A Sasakian manifold $(M, \xi, \eta, \Phi, g)$ of dimension $2 n+1 \geq 5$ admits both the Einstein-Weyl structures $\mathcal{W}^{+}=(g, \theta)$ and $\mathcal{W}^{-}=(g,-\theta)$ for some 1-form $\theta$ if and only if is $\eta$-Einstein with Einstein constants $(\lambda, \nu)$ such that $\nu<0$.

Proof. From the proof of Theorem 60 we know that, if $(M, \xi, \eta, \Phi, g)$ admits both $\mathcal{W}^{ \pm}$as Einstein-Weyl structures, then for the 1-form $\theta$ the relation $\theta(X) \theta(\xi)=0$ holds, for every horizontal vector field $X$ on $M$. On the other hand, since on a Sasakian manifold the Ricci tensor satisfies the equality $\operatorname{Ric}_{g}(\Phi(X), \Phi(Y))=$ $\operatorname{Ric}_{g}(X, Y)-2 n \eta(X) \eta(Y)$, substituting in (35) $\Phi(X)$ for $X$ and $\Phi(Y)$ for $Y$ with $X, Y$ horizontal vector fields, we get

(46) $\operatorname{Ric}_{g}(X, Y)-\frac{s}{2 n+1} g(X, Y)=\frac{2 n-1}{2 n+1}|\theta|^{2} g(X, Y)-(2 n-1) \theta(\Phi(X)) \theta(\Phi(Y))$,

where $s$ is the scalar curvature of $g$. The comparison between (35) and this last equation implies that $\theta$ must also obey the equation

$$
\theta(\Phi(X)) \theta(\Phi(Y))=\theta(X) \theta(Y)
$$

for all horizontal vector fields $X, Y$ on $M$. Now, arguing as in the proof of Theorem [60] if we suppose $\theta(\xi)=\eta\left(\theta^{\#}\right)=0$ and consider $X=Y=\theta^{\#}$ in [47), we obtain $|\theta|=0$ so that $\theta$ identically vanishes on $M$. Finally, the Corollary 62 implies the theorem.

Remark 64. We remark that, by taking Corollary 61 into account, in the case of a compact Sasakian manifold $(M, \xi, \eta, \Phi, g)$, the above Theorem 63 provides the necessary and sufficient condition for $g$ to be the Gauduchon metric of the conformal class $[g]$ for some Einstein-Weyl structure on $M$.

Finally, the following corollary follows easily from the previous analysis.

Corollary 65. Let $(\xi, \eta, \Phi, g)$ be a Sasakian-Einstein structure on a manifold $M$. Then $M$ admits a pair of Einstein-Weyl structures $\mathcal{W}^{ \pm}=(g, \pm \mu \eta)$ obtain by squashing the Sasakian-Einstein structure to an $\eta$-Einstein structure with constants $(\lambda, \nu)$ and $\nu<0$. In this case $\mu^{2}=-\frac{\nu}{2 n-1}$. In particular, all homotopy spheres of dimension $4 n+1,7,11$ and 15 that bound parallelizable manifolds admit many pairs of Einstein-Weyl structures.

The above corollary combined with recent results of BGN03b, BGK03, BGKT03, BG03, Kol04b Kol04a establishes the existence of Einstein-Weyl structures on many other compact simply connected spin manifolds in odd dimensions.

\section{REFERENCES}

[ACGTF04] V. Apostolov, D. M. J. Calderbank, P. Gauduchon, and C. W. TonnesonFriedman, Hamiltonian 2-forms in Kähler geometry, II. Global Classification, arXiv:math.DG/0309408 (2004).

[Aub82] T. Aubin, Nonlinear analysis on manifolds. Monge-Ampère equations, Grundlehren der Mathematischen Wissenschaften [Fundamental Principles of Mathematical Sciences], vol. 252, Springer-Verlag, New York, 1982. MR 85j:58002

[Bai57] W. L. Baily, On the imbedding of $V$-manifolds in projective space, Amer. J. Math. 79 (1957), 403-430. MR 20 \#6538

[Bar65] D. Barden, Simply connected five-manifolds, Ann. of Math. (2) 82 (1965), 365-385. MR 32 \#1714

[Bau00] H. Baum, Twistor and Killing spinors in Lorentzian geometry, Global analysis and harmonic analysis (Marseille-Luminy, 1999), Sémin. Congr., vol. 4, Soc. Math. France, Paris, 2000, pp. 35-52. MR 2002d:53060 
[Bel00] F. A. Belgun, On the metric structure of non-Kähler complex surfaces, Math. Ann. 317 (2000), no. 1, 1-40. MR 2002c:32027

[Bel01] Normal CR structures on compact 3-manifolds, Math. Z. 238 (2001), no. 3, 441-460. MR 2002k:32065

[BG00] C. P. Boyer and K. Galicki, On Sasakian-Einstein geometry, Internat. J. Math. 11 (2000), no. 7, 873-909. MR 2001k:53081

[BG01a] Einstein manifolds and contact geometry, Proc. Amer. Math. Soc. 129 (2001), no. 8, 2419-2430 (electronic). MR 2001m:53071

[BG01b] 느 Einstein metrics in dimension five, J. Differential Geom. 57 (2001), no. 3, 443-463. MR 2003b:53047

[BG03] C. P. Boyer and Galicki, Einstein Metrics on Rational Homology Spheres, arXiv:math.DG/0311355 (2003).

[BGK03] C. P. Boyer, K. Galicki, and J. Kollár, Einstein Metrics on Spheres, to appear in Ann. of Math., arXiv:math.DG/0309408 (2003).

[BGKT03] C. P. Boyer, K. Galicki, J. Kollár, and E. Thomas, Einstein Metrics on Exotic Spheres in Dimensions 7,11, and 15, to appear in Experimental Mathematics, arXiv:math.DG/0311293 (2003).

[BGN03a] C. P. Boyer, K. Galicki, and M. Nakamaye, On positive Sasakian geometry, Geom. Dedicata 101 (2003), 93-102. MR 2017897

[BGN03b] On the geometry of Sasakian-Einstein 5-manifolds, Math. Ann. 325 (2003), no. 3, 485-524. MR 2004b:53061

[BGN03c] , Sasakian geometry, homotopy spheres and positive Ricci curvature, Topology 42 (2003), no. 5, 981-1002. MR 1978045

[Bla02] D. E. Blair, Riemannian geometry of contact and symplectic manifolds, Progress in Mathematics, vol. 203, Birkhäuser Boston Inc., Boston, MA, 2002. MR 2002m:53120

[Boh03] C. Bohle, Killing spinors on Lorentzian manifolds, J. Geom. Phys. 45 (2003), no. 34, 285-308. MR 1952661

[BPVdV84] W. Barth, C. Peters, and A. Van de Ven, Compact complex surfaces, Ergebnisse der Mathematik und ihrer Grenzgebiete (3) [Results in Mathematics and Related Areas (3)], vol. 4, Springer-Verlag, Berlin, 1984. MR 86c:32026

[Bri66] E. Brieskorn, Beispiele zur Differentialtopologie von Singularitäten, Invent. Math. 2 (1966), 1-14. MR 34 \#6788

[CLS90] P. Candelas, M. Lynker, and R. Schimmrigk, Calabi-Yau manifolds in weighted $\mathbf{P}_{4}$, Nuclear Phys. B 341 (1990), no. 2, 383-402. MR 91m:14062

[CP99] D. M. J. Calderbank and H. Pedersen, Einstein-Weyl geometry, Surveys in differential geometry: essays on Einstein manifolds, Surv. Differ. Geom., VI, Int. Press, Boston, MA, 1999, pp. 387-423. MR 2002b:53062

[CT94] J. Cheeger and G. Tian, On the cone structure at infinity of Ricci flat manifolds with Euclidean volume growth and quadratic curvature decay, Invent. Math. 118 (1994), no. 3, 493-571. MR 95m:53051

[EKA90] A. El Kacimi-Alaoui, Opérateurs transversalement elliptiques sur un feuilletage riemannien et applications, Compositio Math. 73 (1990), no. 1, 57-106. MR 91f:58089

[FS90] R. Fintushel and R. J. Stern, Instanton homology of Seifert fibred homology three spheres, Proc. London Math. Soc. (3) 61 (1990), no. 1, 109-137. MR 91k:57029

[Gau95] P. Gauduchon, Structures de Weyl-Einstein, espaces de twisteurs et variétés de type $S^{1} \times S^{3}$, J. Reine Angew. Math. 469 (1995), 1-50. MR 97d:53048

[Gei97] H. Geiges, Normal contact structures on 3-manifolds, Tohoku Math. J. (2) 49 (1997), no. 3, 415-422. MR 98h:53046

[GH78] P. Griffiths and J. Harris, Principles of algebraic geometry, Wiley-Interscience [John Wiley \& Sons], New York, 1978, Pure and Applied Mathematics. MR 80b:14001

[GMSW04a] J. P. Gauntlett, D. Martelli, J. Sparks, and W. Waldram, Sasaki-Einstein metrics on $S^{2} \times S^{3}$, arXiv:hep-th/0403002 preprint, 2004

[GMSW04b] _ A new infinite class of Sasaki-Einstein manifolds, arXiv:hep-th/0403038 preprint, 2004.

[GO98] P. Gauduchon and L. Ornea, Locally conformally Kähler metrics on Hopf surfaces, Ann. Inst. Fourier (Grenoble) 48 (1998), no. 4, 1107-1127. MR 2000g:53088 
[Gra59] J. W. Gray, Some global properties of contact structures, Ann. of Math. (2) 69 (1959), 421-450. MR 22 \#3016

[Gui02] B. S. Guilfoyle, The local moduli of Sasakian 3-manifolds, Int. J. Math. Math. Sci. 32 (2002), no. 2, 117-127. MR 2004f:53049

[Hig93] T. Higa, Weyl manifolds and Einstein-Weyl manifolds, Comment. Math. Univ. St. Paul. 42 (1993), no. 2, 143-160. MR 94h:53059

[HS97] A. D. Hwang and S. R. Simanca, Extremal Kähler metrics on Hirzebruch surfaces which are locally conformally equivalent to Einstein metrics, Math. Ann. 309 (1997), no. 1, 97-106. MR 98f:58056

[IF00] A. R. Iano-Fletcher, Working with weighted complete intersections, Explicit birational geometry of 3-folds, London Math. Soc. Lecture Note Ser., vol. 281, Cambridge Univ. Press, Cambridge, 2000, pp. 101-173. MR 2001k:14089

[JK01] J. M. Johnson and J. Kollár, Kähler-Einstein metrics on log del Pezzo surfaces in weighted projective 3-spaces, Ann. Inst. Fourier (Grenoble) 51 (2001), no. 1, 69-79. MR 2002b:32041

[Kol04a] J. Kollár, Einstein metrics on 5-dimensional Seifert bundles, arXiv:math.DG/0408184 (2004).

[Kol04b] Einstein metrics on connected sums of $S^{2} \times S^{3}$, arXiv:math.DG/0402141 (2004).

[KW99] I. R. Klebanov and E. Witten, Superconformal field theory on threebranes at a Calabi-Yau singularity, Nuclear Phys. B 536 (1999), no. 1-2, 199-218. MR 99k:81253

[Mal99] J. Maldacena, The large- $N$ limit of superconformal field theories and supergravity, Internat. J. Theoret. Phys. 38 (1999), no. 4, 1113-1133, Quantum gravity in the southern cone (Bariloche, 1998). MR 2001h:81246

[MSY05] D. Martelli, J. Sparks, and S.-T. Yau, The geometric dual of a-maximisation for toric Sasaki-Einstein manifolds, arXiv:hep-th/0503183 preprint, 2005.

[Mat00] P. Matzeu, Some examples of Einstein-Weyl structures on almost contact manifolds, Classical Quantum Gravity 17 (2000), no. 24, 5079-5087. MR 2002b:53066

[Mat02] _ Almost contact Einstein-Weyl structures, Manuscripta Math. 108 (2002), no. 3, 275-288. MR 2003g:53065

[Mil75] J. Milnor, On the 3-dimensional Brieskorn manifolds $M(p, q, r)$, Knots, groups, and 3-manifolds (Papers dedicated to the memory of R. H. Fox), Princeton Univ. Press, Princeton, N. J., 1975, pp. 175-225. Ann. of Math. Studies, No. 84. MR 54 \#6169

[MO70] J. Milnor and P. Orlik, Isolated singularities defined by weighted homogeneous polynomials, Topology 9 (1970), 385-393. MR 45 \#2757

[Mor97] A. Moroianu, Parallel and Killing spinors on $\mathrm{Spin}^{c}$ manifolds, Comm. Math. Phys. 187 (1997), no. 2, 417-427. MR 98i:58245

[MS05] D. Martelli and J. Sparks, Toric geometry, Sasaki-Einstein manifolds and a new infinite class of ads/cft duals, Comm. Math. Phys. (2005), to appear; arXiv:hep-th/0411238

[Nar93] F. Narita, Riemannian submersion with isometric reflections with respect to the fibers, Kodai Math. J. 16 (1993), no. 3, 416-427. MR 94f:53105

[Nar97] _ Riemannian submersions and Riemannian manifolds with Einstein-Weyl structures, Geom. Dedicata 65 (1997), no. 1, 103-116. MR 98d:53068

[Nar98] _ Einstein-Weyl structures on almost contact metric manifolds, Tsukuba J. Math. 22 (1998), no. 1, 87-98. MR 99g:53051

[Oku62] M. Okumura, Some remarks on space with a certain contact structure, Tôhoku Math. J. (2) 14 (1962), 135-145. MR 26 \#708

[Orl70] P. Orlik, Weighted homogeneous polynomials and fundamental groups, Topology 9 (1970), 267-273. MR 41 \#6251

[Pet98] P. Petersen, Riemannian geometry, Graduate Texts in Mathematics, vol. 171, Springer-Verlag, New York, 1998. MR 98m:53001

[PS93] H. Pedersen and A. Swann, Riemannian submersions, four-manifolds and EinsteinWeyl geometry, Proc. London Math. Soc. (3) 66 (1993), no. 2, 381-399. MR 94c:53061 
[Rei80] M. Reid, Canonical 3-folds, Journées de Géometrie Algébrique d'Angers, Juillet 1979/Algebraic Geometry, Angers, 1979, Sijthoff \& Noordhoff, Alphen aan den Rijn, 1980, pp. 273-310. MR 82i: 14025

[Sae87] O. Saeki, Knotted homology spheres defined by weighted homogeneous polynomials, J. Fac. Sci. Univ. Tokyo Sect. IA Math. 34 (1987), no. 1, 43-50. MR 88c:57021

[Sas65] S. Sasaki, Almost Contact Manifolds, Part 1, Lecture Notes, Mathematical Institute, Tôhoku University (1965).

[Sas68] - Almost Contact Manifolds, Part 3, Lecture Notes, Mathematical Institute, Tôhoku University (1968).

[Sav02] N. Saveliev, Invariants for homology 3-spheres, Encyclopaedia of Mathematical Sciences, vol. 140, Springer-Verlag, Berlin, 2002, Low-Dimensional Topology, 1. MR 2004c:57026

[Sco83] P. Scott, The geometries of 3-manifolds, Bull. London Math. Soc. 15 (1983), no. 5, 401-487. MR 84m:57009

[Sma62] S. Smale, On the structure of 5-manifolds, Ann. of Math. (2) 75 (1962), 38-46. MR $25 \# 4544$

[Tak78] T. Takahashi, Deformations of Sasakian structures and its application to the Brieskorn manifolds, Tôhoku Math. J. (2) 30 (1978), no. 1, 37-43. MR 81e:53024

[Tan68] S. Tanno, The topology of contact Riemannian manifolds, Illinois J. Math. 12 (1968), 700-717. MR 38 \#2803

[Tan69] - Sasakian manifolds with constant $\phi$-holomorphic sectional curvature, Tôhoku Math. J. (2) 21 (1969), 501-507. MR 40 \#4894

[Tan79] Geodesic flows on $C_{L}$-manifolds and Einstein metrics on $S^{3} \times S^{2}$, Minimal submanifolds and geodesics (Proc. Japan-United States Sem., Tokyo, 1977), NorthHolland, Amsterdam, 1979, pp. 283-292. MR 81g:58027

[Thu97] W. P. Thurston, Three-dimensional geometry and topology. Vol. 1, Princeton Mathematical Series, vol. 35, Princeton University Press, Princeton, NJ, 1997, Edited by Silvio Levy. MR 97m:57016

[Ton97] P. Tondeur, Geometry of foliations, Monographs in Mathematics, vol. 90, Birkhäuser Verlag, Basel, 1997. MR 98d:53037

[Yau78] S.-T. Yau, On the Ricci curvature of a compact Kähler manifold and the complex Monge-Ampère equation. I, Comm. Pure Appl. Math. 31 (1978), no. 3, 339-411. MR 81d:53045

CPB: Department of Mathematics \& Statistics, University of New Mexico, AlbuQUERQUE, NM 87131.

E-mail address: cboyer@math.unm.edu

KG: Max-Planck-Institut für Mathematik, D53111 Bonn, Germany; on leave from Department of Mathematics and Statistics, University of New Mexico, Albuquerque, NM 87131

E-mail address: galicki@math.unm.edu

PM: Dipartimento di Matematica e Informatica, Universitá di Cagliari, Cagliari, ITALY.

E-mail address: matzeu@vaxca1.unica.it 